TITLE:

\title{
Towards a classification of bifurcations in Vlasov equations
}

$\operatorname{AUTHOR}(S)$ :

Barré, J.; Métivier, D.; Yamaguchi, Y. Y.

\section{CITATION:}

Barré, J. ... [et al]. Towards a classification of bifurcations in Vlasov equations. Physical Review E 2020, 102(5): 052208.

ISSUE DATE:

2020-11

URL:

http://hdl.handle.net/2433/258958

RIGHT:

(C)2020 American Physical Society 


\title{
Towards a classification of bifurcations in Vlasov equations
}

\author{
J. Barré \\ Institut Denis Poisson, Université d'Orléans, CNRS, Université de Tours, France \\ and Institut Universitaire de France, Bâtiment de mathématiques, Rue de Chartres, B.P. 6759, 45067, Orléans cedex 2, France \\ D. Métivier \\ Center for Nonlinear Studies and Theoretical Division T-4, Los Alamos National Laboratory, Los Alamos, New Mexico 87544, USA \\ Y. Y. Yamaguchi \\ Department of Applied Mathematics and Physics, Graduate School of Informatics, Kyoto University, Kyoto 606-8501, Japan
}

(Received 25 September 2019; accepted 15 October 2020; published 9 November 2020)

\begin{abstract}
We propose a classification of bifurcations of Vlasov equations, based on the strength of the resonance between the unstable mode and the continuous spectrum on the imaginary axis. We then identify and characterize a new type of generic bifurcation where this resonance is weak, but the unstable mode couples with a stable mode and a Casimir invariant of the system to form a size-3 Jordan block. We derive a three-dimensional reduced noncanonical Hamiltonian system describing this bifurcation. Comparison of the reduced dynamics with direct numerical simulations on a test case gives excellent agreement. We finally discuss the relevance of this bifurcation to specific physical situations.
\end{abstract}

DOI: 10.1103/PhysRevE.102.052208

\section{INTRODUCTION}

A wide variety of physical systems are governed over certain timescales by mean-field forces rather than collisions between their constituents. The appropriate kinetic description is then a Vlasov, or Vlasov-like, equation. In this category we find Vlasov-Poisson equation at the heart of plasma physics, the collisionless Boltzmann equation (or Vlasov-Newton equation) describing self-gravitating systems, but also fluid models such as the two-dimensional Euler equation. Indeed many ideal fluid and MHD models share with Vlasov-Poisson equation a similar infinite dimensional noncanonical Hamiltonian structure [1-4]. Vlasov equations possess both regular features (such as an infinite number of conserved quantities) and chaotic ones (such as the development of infinitely fine structures in phase space) which make both the understanding of their qualitative behavior and their numerical simulation famously difficult problems, relevant in various physical fields. In particular, Vlasov-like equations have an uncountable number of stationary states, and linear and nonlinear stability studies of these states have led, among other important physical and mathematical concepts, to the discovery of Landau damping [5] close to stable stationary states. We are concerned in this article with the question: What happens close to weakly unstable stationary states? This amounts to a study of local bifurcations of Vlasov equations. The rationale is that these bifurcations (1) should be universal, i.e., be relevant for all types of Vlasov equations and (2) could provide basic building blocks to describe the qualitative behavior of these equations.

Vlasov equations are Hamiltonian systems, and while bifurcations in Hamiltonian systems are well known and classified [6-8], the specificities of Vlasov equations bring difficulties. First, their Hamiltonian structure is noncanonical, and highly degenerate [2-4], which is the origin of the infinite number of conserved quantities, called Casimir invariants. We note this degenerate noncanonical structure has been taken advantage of for instance to design numerical schemes in plasma [9-13] and fluid [14,15] contexts, as well as to derive weakly nonlinear expansions [16]. Second, the linearized Vlasov evolution typically features continuous spectrum on the imaginary axis, and a growing unstable mode can create resonances with part of this spectrum, triggering complex dynamics.

The study of Vlasov bifurcations is an old topic. One of the most common Vlasov bifurcations describes the destabilization of a homogeneous stationary state, and is relevant in plasma physics (bump on tail or two-stream instabilities) and fluid dynamics (shear flow instability); the nonlinear development of the instability gave rise to a debate starting in the 1960s [17-20] and concluded in the 1990s, when it became clear that it was governed by resonances. This instability is characterized by strong nonlinear effects, at the origin of "trapping scaling" $[17,21,22]$ : the amplitude of the unstable mode saturates at a level $O\left(\lambda^{2}\right)$, where $\lambda$ is the instability rate, rather than the much larger $O\left(\lambda^{1 / 2}\right)$, as happens for standard supercritical pitchfork or Hopf bifurcations. Furthermore, an infinite dimensional "normal form" has been derived for the near-threshold dynamics, called the Single Wave Model (SWM) [23-25]. It is clear that however important this SWM example may be, it is just one case among many other possible bifurcations, which are much less studied.

As a first example beyond the SWM case, a kind of "very strong" resonance has already been identified in the literature 
TABLE I. Classification sketch for bifurcations in Vlasov-like systems. VSR (SR,WR) represents (very) strong (weak) resonance which occurs without (no-C) or with (with-C) coupling with the Casimir modes at the linear level. SWM represents the single-wave model. SE (RE) represents singular (regular) eigenvectors. The "Scaling" column specifies the perturbation amplitude at which nonlinear effects kick in; $\lambda$ is the instability rate. The fourth line is highlighted as the main subject of this work. (*) See text for the explanation of the $\lambda^{2}$ scaling.

\begin{tabular}{lccccc}
\hline \hline Resonance & Casimir modes & Reduction & Eigenvector & Scaling & Ref. \\
\hline VSR & No-C & See Ref. [28] & SE & $\lambda^{5 / 2}$ & $\lambda^{2}$ \\
SR & No-C & SWM & RE & {$[26-28]$} \\
WR & No-C & Finite dim. & RE & $\lambda^{1 / 2}$ & {$[23]$ Sec. IV A } \\
WR & With-C & Finite dim. & RE & $\lambda^{2}(*)$ & This work \\
\hline \hline
\end{tabular}

[26-28]. The critical eigenvector associated with the instability is in this case singular and entails even stronger nonlinear effects altering the trapping scaling characteristic of the SWM bifurcation: nonlinear effects kick in at an amplitude $O\left(\lambda^{5 / 2}\right)$, to be compared with the $O\left(\lambda^{2}\right)$ for trapping scaling. While this bifurcation was studied only for two-species plasmas, it can likely be found in other contexts as well.

As a second example, which will be the subject of this article, some instabilities do not give rise to resonances, or only to weak ones: physically this mainly corresponds to situations where no or few particles have a frequency close to the frequency of the instability. A weak resonance happens for homogeneous stationary states with some special velocity distributions (e.g., "flat top" velocity distribution; see Ref. [23], Sec. IV A), and, more importantly, this is a generic situation for inhomogeneous stationary states with real bifurcating eigenvalue, as shown in Ref. [29] (the reason for this weak resonance is discussed below). At first sight, it seems that such nonresonant bifurcations can be studied through standard center manifold reduction and hence would fall into the class of normal finite dimensional canonical Hamiltonian bifurcations. While it is essentially true in the "flat top" velocity distribution case [23], we highlight in this article a new type of generic bifurcation for weakly resonant nonoscillatory instabilities, related to the noncanonical Hamiltonian nature of Vlasov equation: a neutral mode associated with a Casimir invariant combines with a stable mode and an unstable mode, thereby forms a three-dimensional Jordan block, and controls the bifurcation. In particular, when the initial perturbation does not modify the Casimir invariants, nonlinear effects kick in when the unstable mode reaches an amplitude $O\left(\lambda^{2}\right)$. However, this scaling can be strongly modified by the Casimir coupling, as we shall explain later, see Fig. 2 and Appendix B. Summarizing the above discussion, Table I sketches a classification of bifurcations in Vlasov-like equations. It is the first product of this article.

We now turn to the main contribution of this work. In Sec. II we provide a brief review of the standard center manifold reduction in a form which is helpful to understand the following discussions. This reduction is applied to a weakly resonant Vlasov bifurcation in Sec. III. This case study shows that the linearized Vlasov equation at the bifurcation point features a three-dimensional Jordan block and that the reduced dynamics at leading nonlinear order is a three dimensional noncanonical Hamiltonian dynamics on a corresponding three-dimensional reduced space. The results obtained in Sec. III are generalized in Sec. IV using a matrix formal expression of the Vlasov equation, capturing the essential points of our discussions. We also reveal that the three-dimensional Hamiltonian dynamics on the reduced space found on the example of Sec. III can be understood as a normal form. In Sec. V, using the model introduced in Sec. III, the reduced dynamics is quantitatively (beyond the scaling mentioned above) compared with direct numerical simulations of the Vlasov equation. Finally, in Sec. VI, we discuss the expected physical applications of this bifurcation. Some tedious computations are gathered in the Appendices to avoid disrupting the main argument of the article.

\section{CENTER MANIFOLD REDUCTION}

We briefly review the center manifold reduction technique. At a marginally stable equilibrium point (the bifurcation point), the linearized dynamics features a center subspace $E_{\mathrm{c}}$, corresponding to the eigenvalues with vanishing real part. The invariant manifold $\mathcal{M}_{\mathrm{c}}$ which generalizes $E_{\mathrm{c}}$ at the nonlinear level is called central manifold. The reduced dynamics on $\mathcal{M}_{\mathrm{c}}$ captures the qualitatively important phenomena at, and close to, the bifurcation point. The goal of this section is to explain this procedure on a very simple example, which, albeit in a finite dimensional setting, will be relevant for the following weakly resonant Vlasov bifurcation.

Let us consider a dynamical system on $\mathbb{R}^{4}$,

$$
\frac{d \boldsymbol{r}}{d t}=\mathcal{L}_{\mu} \cdot \boldsymbol{r}+\boldsymbol{g}(x, y, z, w ; \mu),
$$

where

$$
\boldsymbol{r}=x \boldsymbol{e}_{x}+y \boldsymbol{e}_{y}+z \boldsymbol{e}_{z}+w \boldsymbol{e}_{w}=\left(\begin{array}{lll}
\boldsymbol{e}_{x} & \cdots & \boldsymbol{e}_{w}
\end{array}\right)\left(\begin{array}{c}
x \\
\vdots \\
w
\end{array}\right)
$$

and the basis $\boldsymbol{e}_{x}, \ldots, \boldsymbol{e}_{w}$ is standard. The parameter $\mu$ controls a bifurcation and $\mu=0$ is the critical point. $\mathcal{L}_{\mu} \cdot \boldsymbol{r}$ represents the action of the linear operator $\mathcal{L}_{\mu}$ on the vector $\boldsymbol{r}$ and $\boldsymbol{g}(x, y, z, w ; \mu)$ is of $O_{2}(x, y, z, w)$, where $O_{n}(x, y, z, w)$ represents terms of order $n$ or higher in $x, y, z, w$ and hence the symbol $O_{n}(x)$ means $O\left(x^{n}\right)$ (in other words, both $g$ and its Jacobian matrix with respect to $\boldsymbol{r}$ vanish at the point $\boldsymbol{r}=\mathbf{0}$ ). Clearly, the origin $\boldsymbol{r}=\mathbf{0}$ is an equilibrium point for any $\mu$.

We further expand the linear operator $\mathcal{L}_{\mu}$ as

$$
\mathcal{L}_{\mu}=\mathcal{L}_{0}+\mu \delta \mathcal{L}_{\mu}
$$

and the system (1) is expressed by

$$
\frac{d \boldsymbol{r}}{d t}=\mathcal{L}_{0} \cdot \boldsymbol{r}+\boldsymbol{N}(x, y, z, w ; \mu),
$$


where

$$
\boldsymbol{N}(x, y, z, w ; \mu)=\mu \delta \mathcal{L}_{\mu} \cdot \boldsymbol{r}+\boldsymbol{g}(x, y, z, w ; \mu)
$$

is of $O_{2}(x, y, z, w, \mu)$.

The matrix $\mathbf{L}_{0}$ representing $\mathcal{L}_{0}$ is defined by

$$
\begin{gathered}
\left(\begin{array}{cccc}
\mathcal{L}_{0} \cdot \boldsymbol{e}_{x} & \mathcal{L}_{0} \cdot \boldsymbol{e}_{y} & \mathcal{L}_{0} \cdot \boldsymbol{e}_{z} & \mathcal{L}_{0} \cdot \boldsymbol{e}_{w}
\end{array}\right) \\
=\left(\begin{array}{llll}
\boldsymbol{e}_{x} & \boldsymbol{e}_{y} & \boldsymbol{e}_{z} & \boldsymbol{e}_{w}
\end{array}\right) \mathbf{L}_{0},
\end{gathered}
$$

and we assume

$$
\mathbf{L}_{0}=\left(\begin{array}{cccc}
0 & 1 & 0 & 0 \\
0 & 0 & 1 & 0 \\
0 & 0 & 0 & 0 \\
0 & 0 & 0 & -1
\end{array}\right)
$$

We have chosen $\mathbf{L}_{0}$ with a size-3 Jordan block corresponding to the generalized eigenspace of the eigenvalue 0 at the critical point, as this will be the case later for the Vlasov bifurcation.

The center subspace in this example is

$$
E_{\mathrm{c}}=\operatorname{Span}\left\{\boldsymbol{e}_{x}, \boldsymbol{e}_{y}, \boldsymbol{e}_{z}\right\} .
$$

Further, we introduce an inner product $\langle\cdot, \cdot\rangle$ on $\mathbb{R}^{4}$, which is here assumed to be the standard one. This inner product is necessary to introduce the adjoint operator $\mathcal{L}_{0}^{\dagger}$ of $\mathcal{L}_{0}$, which is defined by $\left\langle\mathcal{L}_{0}^{\dagger} \boldsymbol{w}, \boldsymbol{v}\right\rangle=\left\langle\boldsymbol{w}, \mathcal{L}_{0} \boldsymbol{v}\right\rangle$ for any $\boldsymbol{v}, \boldsymbol{w} \in \mathbb{R}^{4}$, and the representation matrix of $\mathcal{L}_{0}^{\dagger}, \mathbf{L}_{0}^{\dagger}$, is the transposition of $\mathbf{L}_{0}$. We shall now reduce the full dynamics (1) onto a threedimensional space around the origin $\boldsymbol{r}=\mathbf{0}$ and around the critical point $\mu=0$.

\section{A. Linear analysis}

We construct a projection operator $\Pi$ from $\mathbb{R}^{4}$ to $E_{\mathrm{c}}$ :

$$
\Pi \cdot \boldsymbol{r}=\boldsymbol{e}_{x}\left\langle\boldsymbol{e}_{x}, \boldsymbol{r}\right\rangle+\boldsymbol{e}_{y}\left\langle\boldsymbol{e}_{y}, \boldsymbol{r}\right\rangle+\boldsymbol{e}_{z}\left\langle\boldsymbol{e}_{z}, \boldsymbol{r}\right\rangle,
$$

which satisfies $\Pi \cdot(\Pi \cdot \boldsymbol{r})=\Pi \cdot \boldsymbol{r}$. This operator is easily found in this simple example; a systematic way to build it is through the adjoint operator $\mathcal{L}_{0}^{\dagger}$ of $\mathcal{L}_{0}$ and its (generalized) eigenvectors.

Both $\mathcal{L}_{0}$ and $\mathcal{L}_{0}^{\dagger}$ have an eigenvector and two generalized eigenvectors for the eigenvalue 0 :

$$
\begin{aligned}
& \mathcal{L}_{0} \cdot \boldsymbol{e}^{(0)}=\mathbf{0}, \quad \mathcal{L}_{0} \cdot \boldsymbol{e}^{(1)}=\boldsymbol{e}^{(0)}, \quad \mathcal{L}_{0} \cdot \boldsymbol{e}^{(2)}=\boldsymbol{e}^{(1)}, \\
& \left(\boldsymbol{e}^{(0)}=\boldsymbol{e}_{x}, \quad \boldsymbol{e}^{(1)}=\boldsymbol{e}_{y}, \quad \boldsymbol{e}^{(2)}=\boldsymbol{e}_{z}\right)
\end{aligned}
$$

and

$$
\begin{aligned}
& \mathcal{L}_{0}^{\dagger} \cdot \boldsymbol{f}^{(0)}=\mathbf{0}, \quad \mathcal{L}_{0}^{\dagger} \cdot \boldsymbol{f}^{(1)}=\boldsymbol{f}^{(0)}, \quad \mathcal{L}_{0}^{\dagger} \cdot \boldsymbol{f}^{(2)}=\boldsymbol{f}^{(1)}, \\
& \left(\boldsymbol{f}^{(0)}=\boldsymbol{e}_{z}, \quad \boldsymbol{f}^{(1)}=\boldsymbol{e}_{y}, \quad \boldsymbol{f}^{(2)}=\boldsymbol{e}_{x}\right),
\end{aligned}
$$

which reflect the upper-left size-3 Jordan block of $\mathbf{L}_{0}$. From the structure of the scalar products

$$
\left(\begin{array}{ccc}
\left\langle\boldsymbol{f}^{(0)}, \boldsymbol{e}^{(0)}\right\rangle & \left\langle\boldsymbol{f}^{(0)}, \boldsymbol{e}^{(1)}\right\rangle & \left\langle\boldsymbol{f}^{(0)}, \boldsymbol{e}^{(2)}\right\rangle \\
\left\langle\boldsymbol{f}^{(1)}, \boldsymbol{e}^{(0)}\right\rangle & \left\langle\boldsymbol{f}^{(1)}, \boldsymbol{e}^{(1)}\right\rangle & \left\langle\boldsymbol{f}^{(1)}, \boldsymbol{e}^{(2)}\right\rangle \\
\left\langle\boldsymbol{f}^{(2)}, \boldsymbol{e}^{(0)}\right\rangle & \left\langle\boldsymbol{f}^{(2)}, \boldsymbol{e}^{(1)}\right\rangle & \left\langle\boldsymbol{f}^{(2)}, \boldsymbol{e}^{(2)}\right\rangle
\end{array}\right)=\left(\begin{array}{ccc}
0 & 0 & 1 \\
0 & 1 & 0 \\
1 & 0 & 0
\end{array}\right),
$$

the projection operator $\Pi$ is built:

$$
\Pi \cdot \boldsymbol{r}=\boldsymbol{e}^{(0)}\left\langle\boldsymbol{f}^{(2)}, \boldsymbol{r}\right\rangle+\boldsymbol{e}^{(1)}\left\langle\boldsymbol{f}^{(1)}, \boldsymbol{r}\right\rangle+\boldsymbol{e}^{(2)}\left\langle\boldsymbol{f}^{(0)}, \boldsymbol{r}\right\rangle ;
$$

this recovers (9) in this simple example and projects $\mathbb{R}^{4}$ onto the center subspace

$$
E_{\mathrm{c}}=\operatorname{Span}\left\{\boldsymbol{e}^{(0)}, \boldsymbol{e}^{(1)}, \boldsymbol{e}^{(2)}\right\} .
$$

In a Vlasov system at a bifurcating inhomogeneous stationary state, the same structure (12) for (generalized) eigenvectors and projection operator can be found in (46) and in (48) respectively (see Appendix A for general spatially onedimensional systems).

\section{B. Nonlinear analysis}

We now construct amplitude equations, which represent the reduced dynamics on $\mathcal{M}_{\mathrm{c}}$. The center manifold $\mathcal{M}_{c}$ is actually built order by order at the same time the reduced dynamics is found. Suppose that $\mathcal{M}_{c}$ is described, at least locally, as a "graph" over the vector space $E_{c}$; i.e., there exists a function $S$ such that any point $\boldsymbol{r}$ on $\mathcal{M}_{c}$ can be written as

$$
\boldsymbol{r}=A_{0} \boldsymbol{e}^{(0)}+A_{1} \boldsymbol{e}^{(1)}+A_{2} \boldsymbol{e}^{(2)}+S\left(A_{0}, A_{1}, A_{2}\right) \boldsymbol{e}_{w},
$$

where $S\left(A_{0}, A_{1}, A_{2}\right)$ is of $O_{2}\left(A_{0}, A_{1}, A_{2}\right)$ : this reflects the fact that $\mathcal{M}_{c}$ is tangent to $E_{\mathrm{c}}$ at the origin, the equilibrium point.

Assuming now $\boldsymbol{r}$ depends on $t$, the coefficients $A_{i}$ in (15) depend on $t$. Then differentiating (15) with respect to $t$, we have

$$
\begin{aligned}
\frac{d \boldsymbol{r}}{d t}= & \dot{A}_{0} \boldsymbol{e}^{(0)}+\dot{A}_{1} \boldsymbol{e}^{(1)}+\dot{A}_{2} \boldsymbol{e}^{(2)} \\
& +\left(\frac{\partial S}{\partial A_{0}} \dot{A}_{0}+\frac{\partial S}{\partial A_{1}} \dot{A}_{1}+\frac{\partial S}{\partial A_{2}} \dot{A}_{2}\right) \boldsymbol{e}_{w} .
\end{aligned}
$$

Substituting (15) into the equation of motion (4), we also have

$$
\begin{aligned}
\frac{d \boldsymbol{r}}{d t}= & A_{1}(t) \boldsymbol{e}^{(0)}+A_{2}(t) \boldsymbol{e}^{(1)}-S \boldsymbol{e}_{w} \\
& +N\left(A_{0}(t), A_{1}(t), A_{2}(t), S ; \mu\right) .
\end{aligned}
$$

Operating the projection operator (13) on Eqs. (16) and (17), and picking up the coefficients of $\boldsymbol{e}^{(0)}, \boldsymbol{e}^{(1)}$, and $\boldsymbol{e}^{(2)}$, we obtain the amplitude equations at quadratic order:

$$
\begin{aligned}
& \dot{A_{0}}=A_{1}+\left\langle\boldsymbol{f}^{(2)}, N\left(A_{0}, A_{1}, A_{2}, 0 ; \mu\right)\right\rangle+O_{3}\left(A_{0}, A_{1}, A_{2}, \mu\right), \\
& \dot{A_{1}}=A_{2}+\left\langle\boldsymbol{f}^{(1)}, \boldsymbol{N}\left(A_{0}, A_{1}, A_{2}, 0 ; \mu\right)\right\rangle+O_{3}\left(A_{0}, A_{1}, A_{2}, \mu\right), \\
& \dot{A_{2}}=\left\langle\boldsymbol{f}^{(0)}, \boldsymbol{N}\left(A_{0}, A_{1}, A_{2}, 0 ; \mu\right)\right\rangle+O_{3}\left(A_{0}, A_{1}, A_{2}, \mu\right) .
\end{aligned}
$$

We have used here that $\boldsymbol{g}$ is of $O_{2}(x, y, z, w)$ and that $\boldsymbol{e}_{w}$ is orthogonal to $E_{\mathrm{c}}$.

We underline that the function $S$ does not contribute to these amplitude equations at quadratic order. In other words, up to quadratic order in the amplitude equations, approximating $\mathcal{M}_{\mathrm{c}}$ by $E_{\mathrm{c}}$ is enough: this will be helpful later. Going beyond quadratic order requires expanding the nonlinearity $g$, and to compute order by order the graph of the center manifold $S$; this is possible by operating Id $-\Pi$ on (16) and (17).

We summarize three important remarks gathered from this simple example: (1) The projection operator is constructed through computing (generalized) eigenvectors for the eigenvalue 0 of the linear operator $\mathcal{L}_{0}$ and its adjoint $\mathcal{L}_{0}^{\dagger}$. (2) The reduced dynamics at quadratic order is obtained by simply projecting the full dynamics on the center subspace $E_{\mathrm{c}}$. (3) The reduced dynamics has a parameter which is constant at 
linear order: $A_{2}$; it will actually be upgraded to a true constant of motion, trace of the Casimirs, in the Vlasov case.

In the following we will analyze Vlasov equations using notations similar to the ones of this section. The spirit of the computations will be the same, but the setting becomes infinite dimensional.

\section{BIFURCATION OF INHOMOGENEOUS STATIONARY STATES OF THE VLASOV EQUATION: AN EXAMPLE}

We now apply these ideas to a bifurcation of Vlasov equation, keeping in mind the three remarks emphasized at the end of Sec. II. Let us recall that looking for a finite dimensional center manifold reduction for the bifurcation of a homogeneous stationary state of Vlasov equation is famously known to be a bad idea [21-23], because of resonances between the destabilizing eigenvector and the continuous spectrum. We will see that the situation is fundamentally different for inhomogeneous stationary states; see also Ref. [29].

\section{A. Specifying the problem}

Vlasov equation describes the evolution of the phase space density $F(\boldsymbol{q}, \boldsymbol{p}, t)$, where $(\boldsymbol{q}, \boldsymbol{p}) \in \mathbb{R}^{n} \times \mathbb{R}^{n}$ are positionmomentum variables:

$$
\begin{aligned}
\frac{\partial F}{\partial t}+\{H[F], F\} & =0 \\
\text { with } H[F](\boldsymbol{q}, \boldsymbol{p}, t) & =\frac{\boldsymbol{p}^{2}}{2}+V[F](\boldsymbol{q}, t) \\
\text { and } V[F](\boldsymbol{q}, t) & =\iint v\left(\boldsymbol{q}-\boldsymbol{q}^{\prime}\right) F\left(\boldsymbol{q}^{\prime}, \boldsymbol{p}^{\prime}, t\right) d \boldsymbol{q}^{\prime} d \boldsymbol{p}^{\prime},
\end{aligned}
$$

$v(\boldsymbol{q})$ is the two-body interaction potential, $V[F]$ is the meanfield potential created by the phase space distribution $F$, and $\{\cdot, \cdot\}$ is the Poisson bracket defined by

$$
\{g, f\}=\left(\nabla_{p} g\right)\left(\nabla_{q} f\right)-\left(\nabla_{q} g\right)\left(\nabla_{p} f\right) .
$$

We introduce an inner product $\langle G, F\rangle$ as

$$
\langle G, F\rangle=\iint G^{*}(\boldsymbol{q}, \boldsymbol{p}) F(\boldsymbol{q}, \boldsymbol{p}) d \boldsymbol{q} d \boldsymbol{p},
$$

where $G^{*}(\boldsymbol{q}, \boldsymbol{p})$ is the complex conjugate of $G(\boldsymbol{q}, \boldsymbol{p})$.

The Vlasov equation (19) has an infinite number of Casimir invariant functionals of the type

$$
\mathcal{C}[F]=\iint \varphi[F(\boldsymbol{q}, \boldsymbol{p}, t)] d \boldsymbol{q} d \boldsymbol{p},
$$

for any smooth function $\varphi$. The existence of the Casimir invariants does not depend on the Hamiltonian $H$ : rather, they come from the degenerate noncanonical Hamiltonian structure of the Vlasov equation [2,3], which will be important to have the size- 3 Jordan block.

Any phase space distribution $F_{\text {stat }}$ of the form

$$
F_{\text {stat }}(\boldsymbol{q}, \boldsymbol{p})=\widehat{F}(h(\boldsymbol{q}, \boldsymbol{p})), \quad h(\boldsymbol{q}, \boldsymbol{p})=\frac{\boldsymbol{p}^{2}}{2}+V\left[F_{\text {stat }}\right](\boldsymbol{q})
$$

is a stationary state of (19). If $V\left[F_{\text {stat }}\right]$ truly depends on the space variables $\boldsymbol{q}, F_{\text {stat }}$ is then a stationary state which is inhomogeneous in space.
Our goal is to study the bifurcation of a family $\left\{F_{\mu}\right\}$ of such inhomogeneous stationary states by varying the parameter $\mu$ : stable for $\mu<0$, unstable for $\mu>0$, and critical for $\mu=0$. The stability change of $F_{\mu}$ is studied by linearizing Vlasov equation (19) around $F_{\mu}$. The spectrum is contained in the imaginary axis for $\mu<0$, and an eigenvalue $\lambda$ leaves the imaginary axis to enter the $\operatorname{Re}(\lambda)>0$ half plane for $\mu>0$. We require this to happen at $\lambda=0$ (steady state bifurcation).

For simplicity, in this section, we use the simplest possible example, the one-dimensional Vlasov equation with $v(q)=$ $-\cos q(q \in[0,2 \pi[)$. The $N$-body system having this interaction is called the Hamiltonian mean-field (HMF) model. The corresponding Vlasov equation is sometimes called the Vlasov-HMF model and has been extensively used as a toy model to explore the intricate dynamics of the Vlasov equation [30-32].

The Vlasov-HMF equation reads

$$
\frac{\partial F}{\partial t}+\left\{H_{\mathrm{HMF}}[F], F\right\}=0
$$

where

$$
H_{\mathrm{HMF}}[F]=\frac{p^{2}}{2}+V_{\mathrm{HMF}}[F]
$$

and

$$
\begin{aligned}
& V_{\mathrm{HMF}}[F](q, t)=-M_{x}[F](t) \cos q-M_{y}[F](t) \sin q, \\
& \text { with } M_{x}[F](t)=\int_{-\infty}^{\infty} d p \int_{0}^{2 \pi} d q \cos q F(q, p, t), \\
& \text { and } M_{y}[F](t)=\int_{-\infty}^{\infty} d p \int_{0}^{2 \pi} d q \sin q F(q, p, t) .
\end{aligned}
$$

The HMF model can be viewed as a model of a ferromagnetic material, and $M_{x}$ and $M_{y}$ are respectively the $x$ and $y$ components of the "magnetization." We expand $F$ around the stationary state $F_{\mu}$ as $F=F_{\mu}+f$. Substituting this expansion into the Vlasov-HMF equation, we have

$$
\frac{\partial f}{\partial t}=\mathcal{L}_{\mu} \cdot f+\mathcal{B}(f, f),
$$

where $\mathcal{L}_{\mu}$ is the linear operator whose action on $f$ is defined by

$$
\mathcal{L}_{\mu} \cdot f=\left\{f, H_{\mathrm{HMF}}\left[F_{\mu}\right]\right\}+\left\{F_{\mu}, V_{\mathrm{HMF}}[f]\right\}
$$

and $\mathcal{B}(f, f)$ is the quadratic operator defined by

$$
\mathcal{B}(g, f)=\left\{g, V_{\mathrm{HMF}}[f]\right\} .
$$

Note that (26) is not a linearized equation but the exact equation including all terms. We further expand the linear operator $\mathcal{L}_{\mu}$ around $\mu=0$ as

$$
\mathcal{L}_{\mu}=\mathcal{L}_{0}+\mu \delta \mathcal{L}_{\mu}
$$

where $\mathcal{L}_{0}$ is the linear operator at the critical point $\mu=0$, and $\delta \mathcal{L}_{\mu}$ may depend on $\mu$. Comparing with the simple example (1), $\mathcal{L}_{0} \cdot f$ plays a role of $\mathcal{L}_{0} \cdot \boldsymbol{r}$ and $\mu \delta \mathcal{L}_{\mu} \cdot f+\mathcal{B}(f, f)$ plays a role of $\mu \delta \mathcal{L}_{\mu} \cdot \boldsymbol{r}+\boldsymbol{g}(x, y, z, w ; \mu)$.

We will show explicitly that the linearized Vlasov operator at the critical stationary state $F_{0}, \mathcal{L}_{0}$, has a three-dimensional Jordan block structure, and that, contrary to the homogeneous case, the resonance with the continuous spectrum is 
weak. We then compute the amplitude equations on the threedimensional reduced space and cast them in a noncanonical Hamiltonian form, which will be easy to compare with the results of the general approach developed in Sec. IV.

\section{B. Linear structure}

Our first task is to study the linearized dynamics around the critical stationary state $F_{0}$,

$$
\frac{\partial f}{\partial t}=\mathcal{L}_{0} \cdot f .
$$

We assume that $F_{0}$ can be written under the form

$$
\begin{aligned}
& F_{0}(q, p)=\widehat{F_{0}}\left(h_{0}(q, p)\right), \\
& h_{0}(q, p)=\frac{p^{2}}{2}-M_{x}\left[F_{0}\right] \cos q-M_{y}\left[F_{0}\right] \sin q,
\end{aligned}
$$

for some function $\widehat{F}_{0}$. From rotational symmetry of the system, we may set $M_{y}\left[F_{0}\right]=0$ without loss of generality. Since we are interested in inhomogeneous stationary states, we assume $M_{x}\left[F_{0}\right] \neq 0$.

The one particle Hamiltonian $h_{0}$ is integrable (it is a simple pendulum Hamiltonian), hence we can introduce the associated angle-action variables $(\theta, J) . h_{0}$ is a function of $J$, so that we can write the stationary state under the form $F_{0}(J)$ (with a slight abuse of notation). The change of variables $(q, p) \rightarrow$ $(\theta, J)$ is canonical, and the Poisson bracket is rewritten as

$$
\{g, f\}=\frac{\partial g}{\partial J} \frac{\partial f}{\partial \theta}-\frac{\partial g}{\partial \theta} \frac{\partial f}{\partial J} .
$$

The linear operator $\mathcal{L}_{0}$ reads

$$
\mathcal{L}_{0} \cdot f=-\Omega_{0}(J) \frac{\partial f}{\partial \theta}+F_{0}^{\prime}(J) \frac{\partial V[f]}{\partial \theta},
$$

where $\Omega_{0}(J)$ is the frequency of a trajectory with action $J$ defined by $\Omega_{0}=d h_{0} / d J$.

We assume that $\mathcal{L}_{0}$ has 0 as eigenvalue, we denote by $\psi^{(0)}$ the corresponding eigenvector, and we look for generalized eigenvectors:

$$
\mathcal{L}_{0} \cdot \psi^{(0)}=0, \quad \mathcal{L}_{0} \cdot \psi^{(j)}=\psi^{(j-1)}, \quad(j \geqslant 1) .
$$

Expanding $\psi^{(j)}(j=0,1,2)$ in Fourier series as

$$
\psi^{(j)}(\theta, J)=\sum_{\alpha \in \mathbb{Z}} \widetilde{\psi}_{\alpha}^{(j)}(J) e^{i \alpha \theta},
$$

we find that $\psi^{(1)}$ and $\psi^{(2)}$ exist, and

$$
\begin{gathered}
\psi^{(0)}=\left(\begin{array}{c}
0 \\
-\frac{F_{0}^{\prime}(J)}{\Omega_{0}(J)} C_{\alpha}(J)
\end{array}\right), \\
\psi^{(1)}=\left(\begin{array}{c}
F_{0}^{\prime}(J) \\
\frac{i \alpha \Omega_{0}^{2}(J)}{} C_{\alpha}(J)
\end{array}\right), \\
\psi^{(2)}=\left(\begin{array}{c}
\widetilde{\psi}_{0}^{(2)}(J) \\
-\frac{F_{0}^{\prime}(J)}{(i \alpha)^{2} \Omega_{0}^{3}(J)} C_{\alpha}(J)
\end{array}\right) .
\end{gathered}
$$

In the vectorial notation above, the first line represents the $\alpha=0$ Fourier component $\widetilde{\psi}_{0}^{(j)}(J)$, and the second one is the general expression for all $\alpha \neq 0$ components. The family of functions $\left\{C_{\alpha}(J)\right\}$ is defined by

$$
C_{\alpha}(J)=\frac{1}{2 \pi} \int_{0}^{2 \pi} \cos q(\theta, J) e^{-i \alpha \theta} d \theta .
$$

$\widetilde{\psi}_{0}^{(2)}(J) \neq 0$ is a partially undetermined function, see Remark 4 below and (A16). There is no $\psi^{(3)}$ satisfying $\mathcal{L}_{0} \cdot \psi^{(3)}=$ $\psi^{(2)}$. Furthermore, the (generalized) eigenvectors above can be normalized such that

$$
\begin{aligned}
& M_{x}\left[\psi^{(0)}\right]=1, \quad M_{x}\left[\psi^{(1)}\right]=M_{x}\left[\psi^{(2)}\right]=0, \\
& M_{y}\left[\psi^{(j)}\right]=0 \quad(j=0,1,2) .
\end{aligned}
$$

These three (generalized) eigenvectors correspond to a size-3 Jordan block for $\mathcal{L}_{0}$ on the subspace $E_{0}=$ $\operatorname{Span}\left\{\psi^{(0)}, \psi^{(1)}, \psi^{(2)}\right\}$ as

$$
\left(\mathcal{L}_{0} \cdot \psi^{(0)} \quad \mathcal{L}_{0} \cdot \psi^{(1)} \quad \mathcal{L}_{0} \cdot \psi^{(2)}\right)=\left(\psi^{(0)} \quad \psi^{(1)} \quad \psi^{(2)}\right) \mathbf{L}_{0},
$$

where

$$
\mathbf{L}_{0}=\left(\begin{array}{lll}
0 & 1 & 0 \\
0 & 0 & 1 \\
0 & 0 & 0
\end{array}\right)
$$

The full Vlasov dynamics will be projected onto this subspace $E_{0}$ as performed in Sec. II.

We progress to study the adjoint operator $\mathcal{L}_{0}^{\dagger}$ of $\mathcal{L}_{0}$, which is written as

$$
\mathcal{L}_{0}^{\dagger} \cdot g=\Omega_{0}(J) \frac{\partial g}{\partial \theta}-V\left[F_{0}^{\prime}(J) \frac{\partial g}{\partial \theta}\right]
$$

from the definition $\left\langle\mathcal{L}_{0}^{\dagger} g, f\right\rangle=\left\langle g, \mathcal{L}_{0} f\right\rangle(\forall f, g)$. Similarly to $\mathcal{L}_{0}$, we find the size-3 Jordan block

$$
\left(\mathcal{L}_{0}^{\dagger} \cdot \phi^{(0)} \quad \mathcal{L}_{0}^{\dagger} \cdot \phi^{(1)} \quad \mathcal{L}_{0}^{\dagger} \cdot \phi^{(2)}\right)=\left(\begin{array}{lll}
\phi^{(0)} & \phi^{(1)} & \phi^{(2)}
\end{array}\right) \mathbf{L}_{0},
$$

where explicit forms of $\phi^{(0)}, \phi^{(1)}$, and $\phi^{(2)}$ are

$$
\begin{aligned}
& \phi^{(0)}=\left(\begin{array}{c}
C_{0}(J) \\
0
\end{array}\right), \quad \phi^{(1)}=\left(\begin{array}{c}
0 \\
\frac{1}{i \alpha \Omega_{0}(J)} C_{\alpha}(J)
\end{array}\right), \\
& \phi^{(2)}=\left(\begin{array}{c}
\widetilde{\phi}_{0}^{(2)}(J) \\
\frac{1}{\left[i \alpha \Omega_{0}(J)\right]^{2}} C_{\alpha}(J)
\end{array}\right),
\end{aligned}
$$

and $\widetilde{\phi}_{0}^{(2)}(J) \propto C_{0}(J)$; see (A3) for details.

The inner products between (generalized) eigenvectors are, as in (12),

$$
\left(\begin{array}{ccc}
\left\langle\phi^{(0)}, \psi^{(0)}\right\rangle & \left\langle\phi^{(0)}, \psi^{(1)}\right\rangle & \left\langle\phi^{(0)}, \psi^{(2)}\right\rangle \\
\left\langle\phi^{(1)}, \psi^{(0)}\right\rangle & \left\langle\phi^{(1)}, \psi^{(1)}\right\rangle & \left\langle\phi^{(1)}, \psi^{(2)}\right\rangle \\
\left\langle\phi^{(2)}, \psi^{(0)}\right\rangle & \left\langle\phi^{(2)}, \psi^{(1)}\right\rangle & \left\langle\phi^{(2)}, \psi^{(2)}\right\rangle
\end{array}\right)=\left(\begin{array}{ccc}
0 & 0 & \star \\
0 & \star & 0 \\
\star & 0 & 0
\end{array}\right),
$$

where the $\star$ are nonzero elements and could be chosen to be 1 with an appropriate normalization of the (generalized) eigenvectors. Note again that the change of variables $(q, p) \rightarrow$ $(\theta, J)$ is canonical, and the inner product is simply transformed as

$$
\langle G, F\rangle=\iint G^{*}(\theta, J) F(\theta, J) d \theta d J
$$


After the normalization, we have a projection operator onto $E_{0}=\operatorname{Span}\left\{\psi^{(0)}, \psi^{(1)}, \psi^{(2)}\right\}:$

$$
\Pi \cdot f=\sum_{j=0}^{2} \psi^{(j)}\left\langle\phi^{(2-j)}, f\right\rangle,
$$

which has the same structure as (13). In Appendix A, the computation is performed for a generic interaction potential $v(q)$ in a spatially one-dimensional system: it is very similar.

Remark 1 . We emphasize the specific role played by the $\alpha=0$ Fourier mode. It corresponds to perturbations $f(\theta, J)$ that depend only on the action $J$ : these are perturbations that modify the values of the Casimir invariants already at linear order since, for $F=F_{0}+f$,

$$
\begin{aligned}
\mathcal{C}[F]-\mathcal{C}\left[F_{0}\right] & =\iint\left(\varphi[F(\theta, J)]-\varphi\left[F_{0}(J)\right]\right) d \theta d J \\
& \simeq \iint \varphi^{\prime}\left[F_{0}(J)\right] f(\theta, J) d \theta d J \\
& =2 \pi \iint \varphi^{\prime}\left[F_{0}(J)\right] \tilde{f}_{0}(J) d J .
\end{aligned}
$$

We also note that there are an infinite number of eigenvectors with eigenvalue 0 , obtained by choosing a nonvanishing zeroth Fourier mode $\widetilde{\psi}_{0}^{(0)}(J)$ in $(36)$; however, nonzero $\widetilde{\psi}_{0}^{(0)}(J)$ are not associated with generalized eigenvectors and a Jordan block. This infinite number of eigenvectors is expected in view of the infinite number of conservation laws. Similarly, there are also eigenvectors associated with a nonzero $M_{y}$.

Remark 2. $\Omega_{0}(J)$ is the frequency of a pendulum: it vanishes at $J=J^{*}$ the action of the separatrix (which separate rotating and librating orbits) in our inhomogeneous setting, inducing a singularity in the expressions of (generalized) eigenvectors. This signals the resonance between the frequency of the marginally stable mode (which is 0 ) and the frequency of the trajectories of some particles (those close to the separatrix). However, $\Omega_{0}$ vanishes only as $-1 / \ln \left|J-J^{*}\right|$ close to the separatrix; as a consequence, all inner products $\left\langle\phi^{(j)}, \psi^{(i)}\right\rangle$ involve only well-defined $J$ integrals irrespective of the power of $\Omega_{0}$ in denominators. This is the mathematical manifestation of the weak resonance for this bifurcation. This weak resonance makes all the difference with the case of a homogeneous marginally stable Vlasov stationary state [29]: the action $J$ is in this case replaced by the momentum $p$, and $1 / \Omega_{0}(J)$ is replaced by $1 / p$, which yields ill-defined $p$ integrals. In particular, the projection operator $\Pi$ is well defined in the inhomogeneous case, and we expect that the reduced dynamics quantitatively approximates the full dynamics. This finite dimensional quantitative approximation is in sharp contrast with the homogeneous case: there, due to strong resonance and pinching singularities, a finite dimensional reduced dynamics provides at best a qualitative picture [22] and an infinite dimensional reduced dynamics is needed [25].

Remark 3. In the Vlasov equation, the linear operator $\mathcal{L}_{0}$ has a continuous spectrum on the imaginary axis, but we shall concentrate on the eigenspace $E_{0}$ instead of the whole (infinite dimensional) center subspace $E_{\mathrm{c}}$. Indeed, thanks to the weak resonance, the eigenvalue 0 can be thought of as effectively isolated from the continuous spectrum: physically, the number of resonant particles is small. However, it is not obvious how to translate this physical intuition into a rigorous mathematical analysis. Hence the validity of our reduction will be confirmed by the successful comparison with numerical simulations in Sec. V.

Remark 4. The arbitrariness in the choice of $\widetilde{\psi}_{0}^{(2)}(J)$ is an advantage for our purposes because it increases the number of initial perturbations that can be accurately represented by their projection onto the three-dimensional subspace $E_{0}$. Indeed, for any initial perturbation which is a linear combination of $\psi^{(0)}, \psi^{(1)}$, and some $\psi^{(2)}$, we can choose this $\psi^{(2)}$ to define $E_{0}$ (which then depends on the initial perturbation). See also the discussion at the end of Sec. A2. We note that $\widetilde{\phi}_{0}^{(2)}(J)$ is restricted by the orthogonal condition $\left\langle\widetilde{\phi}_{0}^{(2)}, \widetilde{\psi}_{0}^{(2)}\right\rangle=0$ once $\widetilde{\psi}_{0}^{(2)}$ is chosen.

\section{Nonlinear analysis: Amplitude equations}

The generalized eigenspace $E_{0}$ is invariant for the linearized dynamics at $\mu=0$. Following Sec. II B, it is natural to look for an invariant manifold $\mathcal{M}_{0}$, tangent to the threedimensional subspace $E_{0}$, on which we can define a reduced dynamics. We are interested in the regime $\mu>0$ and small, when there is a single small unstable eigenvalue.

Starting from the exact Vlasov equation

$$
\frac{\partial f}{\partial t}=\mathcal{L}_{0} \cdot f+\mu \delta \mathcal{L}_{\mu} \cdot f+\mathcal{B}(f, f),
$$

we now have all necessary tools to construct the reduced amplitude equations. Expanding $f \in \mathcal{M}_{0}$ into the form

$$
f(\theta, J, t)=\sum_{i=0}^{2} A_{i}(t) \psi^{(i)}+O_{2}\left(A_{0}, A_{1}, A_{2}, \mu\right)
$$

and recalling the comments after (29), we obtain the amplitude equations for $A_{i}$ :

$$
\begin{aligned}
& \dot{A_{0}}=A_{1}+\left\langle\phi^{(2)}, \mu \delta \mathcal{L}_{\mu} \cdot f\right\rangle+\left\langle\phi^{(2)}, \mathcal{B}(f, f)\right\rangle, \\
& \dot{A_{1}}=A_{2}+\left\langle\phi^{(1)}, \mu \delta \mathcal{L}_{\mu} \cdot f\right\rangle+\left\langle\phi^{(1)}, \mathcal{B}(f, f)\right\rangle, \\
& \dot{A_{2}}=\left\langle\phi^{(0)}, \mu \delta \mathcal{L}_{\mu} \cdot f\right\rangle+\left\langle\phi^{(0)}, \mathcal{B}(f, f)\right\rangle,
\end{aligned}
$$

up to quadratic order. Remember that the $O_{2}\left(A_{0}, A_{1}, A_{2}, \mu\right)$ part in (51) represents the deviation of $\mathcal{M}_{0}$ from $E_{0}$, and does not contribute to the above equations as remarked in Sec. II.

We introduce the coefficients

$$
C_{i j k}=\left\langle\phi^{(i)}, \mathcal{B}\left(\psi^{(j)}, \psi^{(k)}\right)\right\rangle, \quad 0 \leqslant i, j, k \leqslant 2 .
$$

We can see that many coefficients vanish in the Vlasov-HMF case and the nonzero ones are $C_{100}, C_{210}, C_{120}$, and $C_{010}$. We also need to compute the coefficients $\left\langle\phi^{(i)}, \delta \mathcal{L} \cdot \psi^{(j)}\right\rangle$ and the nonzero ones are

$$
\begin{aligned}
& a=\left\langle\phi^{(1)}, \delta \mathcal{L}_{\mu} \cdot \psi^{(0)}\right\rangle, \quad b=\left\langle\phi^{(2)}, \delta \mathcal{L}_{\mu} \cdot \psi^{(1)}\right\rangle, \\
& c=\left\langle\phi^{(1)}, \delta \mathcal{L}_{\mu} \cdot \psi^{(2)}\right\rangle .
\end{aligned}
$$

The amplitude equations up to quadratic order (52) then simplify

$$
\begin{aligned}
& \dot{A_{0}}=(1+\mu b) A_{1}+C_{210} A_{0} A_{1}, \\
& \dot{A_{1}}=(1+\mu c) A_{2}+\mu a A_{0}+C_{100} A_{0}^{2}+C_{120} A_{0} A_{2}, \\
& \dot{A_{2}}=C_{010} A_{0} A_{1},
\end{aligned}
$$

where the coefficients have to be computed numerically. 


\section{Hamiltonian form}

We perform successively the near identity changes of variables

$$
\begin{aligned}
& X_{0}=A_{0}-\left(\frac{1}{2} C_{210}+\frac{1}{6} C_{120}\right) A_{0}^{2}, \\
& X_{1}=A_{1}-\frac{1}{3} C_{120} A_{0} A_{1}, \\
& X_{2}=A_{2}+C_{100} A_{0}^{2}-\frac{1}{3} C_{120} A_{1}^{2}+\frac{2}{3} C_{120} A_{0} A_{2},
\end{aligned}
$$

then

$$
\begin{aligned}
& Y_{0}=\frac{1}{(1+\mu c)(1+\mu b)} X_{0} \\
& Y_{1}=\frac{1}{1+\mu c} X_{1} \\
& Y_{2}=X_{2}+\mu a Y_{0}
\end{aligned}
$$

and finally

$$
\begin{aligned}
Q & =Y_{0} \\
P & =Y_{1} \\
Z & =Y_{2}-\frac{1}{2} r Y_{0}^{2}-\mu a Y_{0}
\end{aligned}
$$

with $r=C_{010}+2 C_{100}$. Truncating the equations of motion at quadratic order, and neglecting terms of $O_{3}(Q, P, Z, \mu)$, they become

$$
\begin{aligned}
& \dot{Q}=P \\
& \dot{P}=Z+\frac{1}{2} r Q^{2}+\mu a Q . \\
& \dot{Z}=0
\end{aligned}
$$

This can be rewritten as a noncanonical Hamiltonian system, with degenerate Poisson operator:

$$
\dot{U}=\mathbf{J} \nabla H(U),
$$

with

$$
\begin{gathered}
U=\left(\begin{array}{c}
Q \\
P \\
Z
\end{array}\right), \quad \mathbf{J}=\left(\begin{array}{ccc}
0 & 1 & 0 \\
-1 & 0 & 0 \\
0 & 0 & 0
\end{array}\right), \\
H(U)=\frac{1}{2} P^{2}-\frac{1}{6} r Q^{3}-\frac{1}{2} \mu a Q^{2}-Q Z .
\end{gathered}
$$

The noncanonical Hamiltonian system (60) is our final reduced system. Notice that at linear order, $Q, P$, and $Z$ are directly proportional to $A_{0}, A_{1}$, and $A_{2}$, respectively, the coefficients of the eigenvector $\psi^{(0)}$ and the generalized eigenvectors $\psi^{(1)}$ and $\psi^{(2)}$. Hence $Q$ essentially measures the amplitude of the magnetization, and $Z$ the change in the Casimir invariants induced by the initial perturbation (the value of $Z$ is determined depending on the selection of $\psi^{(2)}$ as mentioned in Remark 4). In particular, in this Vlasov-HMF case, we will see that $Q$ is directly related to the magnetization of the system. Another useful remark is that the reduced Hamiltonian system (60) has only two parameters: $r$ and $a$, and $r$ depends only on $C_{010}$ and $C_{100}$. Thus, in order to simulate (60) for the VlasovHMF case, we do not need to compute the other nonzero coefficients appearing in the amplitude equations (55). In a general case, from the normal form obtained in Sec. IV B, we hypothesize that the reduced system keeps the form of (60) but the coefficients $a$ and $r$ may depend on the choice of $\psi^{(2)}$. It is also worth mentioning that the solution to Eq. (59) can be expressed in terms of the Weierstrass elliptic function; see Appendix B.

\section{FORMAL NONCANONICAL HAMILTONIAN SYSTEM}

The above computation was performed for a specific onedimensional Vlasov model with periodic boundary conditions for clarity. We obtained a size-3 Jordan block (42) in the linear analysis, and a three-dimensional noncanonical Hamiltonian system (60) at leading nonlinear order. We now derive the same results for a generic noncanonical Hamiltonian system, using a matrix formalism: this allows us to pinpoint the essential points of the mechanism, and emphasize that they generically occur in Vlasov systems at a weakly resonant bifurcation; hence it suggests this bifurcation is indeed generic for Vlasov systems.

\section{A. Size-3 Jordan block structure}

We first prove that a noncanonical Hamiltonian system linearized around a critical point generically has a size-3 Jordan block. The proof below is essentially an abstraction of the explicit computations of Sec. III. The key ingredients are the following [2,3]: (1) Eq. (19) can be seen as an infinite dimensional noncanonical Hamiltonian system and (2) Casimir conservation directly stems from the degeneracy of this structure. Furthermore, the absence (or weakness) of resonances between the bifurcating eigenvalue and the particles frequencies means that the infinite dimensionality plays no role in the three-dimensional Jordan block structure at the bifurcation point. Hence, to make our point clearer, we will use a matrix formal representation of Vlasov equation. A noncanonical Hamiltonian system reads

$$
\dot{y}=\mathbf{J}(y) \nabla H(y),
$$

where $\mathbf{J}$ is a degenerate Poisson matrix, depending on the state $y$, and $H$ is the Hamiltonian. For the Vlasov equation, $y$ would be the density function $F$ over phase space, the gradient $\nabla$ a functional derivative, and $\mathbf{J}$ an operator. We call $y_{0}$ the critical stationary state around which we want to linearize (61); hence $\nabla H\left(y_{0}\right) \in \operatorname{Ker} \mathbf{J}\left(y_{0}\right)$. We assume that $y_{0}$ is not singular for $\mathbf{J}$, i.e., $\mathbf{J}$ has a constant rank in a neighborhood of $y_{0}$.

Weinstein's splitting theorem [33] implies that, up to a local coordinate change, the Poisson operator can be written as

$$
\mathbf{J}=\left(\begin{array}{cc}
\mathbf{J}_{0} & 0_{2 n, m} \\
0_{m, 2 n} & 0_{m, m}
\end{array}\right), \quad \mathbf{J}_{0}=\left(\begin{array}{cc}
0_{n, n} & \mathbf{I}_{n} \\
-\mathbf{I}_{n} & 0_{n, n}
\end{array}\right) .
$$

Here $0_{k, l}$ is the zero matrix of size $k \times l$ and $\mathbf{I}_{n}$ is the unit matrix of size $n$. The appropriate change of variable transforming the operator $\mathbf{J}$ to this simplified form can be built order by order (see Ref. [16]), where the procedure is called "beatification." In practice, we will need only the lowest order. 
The degenerate part, $0_{m, m}$ in $\mathbf{J}$, corresponds to the linearized Casimir invariants $z$ with the notation $y=(x, z)(x \in$ $\left.\mathbb{R}^{2 n}, z \in \mathbb{R}^{m}\right)$. This part makes $\mathbf{J}$ not invertible: in particular we may have $\nabla H\left(y_{0}\right) \neq 0_{2 n+m}$, where $0_{2 n+m}$ is the origin of $\mathbb{R}^{2 n+m}$, even at the stationary point $y_{0}$. However we know $\nabla H\left(y_{0}\right)$ belongs to the kernel of $\mathbf{J}$, which is spanned by the linearized Casimir invariants; hence we may assume $\nabla H\left(y_{0}\right)=0_{2 n+m}$ by adding to $H$ a linear combination of the Casimir invariants. The linearized equation around $y_{0}$ is, therefore, $\dot{\eta}=\mathbf{L} \eta$, where $\eta=y-y_{0}, \mathbf{L}=\mathbf{J S}$, and $\mathbf{S}$ is the Hessian matrix of $H$ at $y_{0}$. We write

$$
\mathbf{S}=\left(\begin{array}{ll}
\mathbf{S}_{x x} & \mathbf{S}_{x z} \\
\mathbf{S}_{z x} & \mathbf{S}_{z z}
\end{array}\right)
$$

where $\mathbf{S}_{x x} \in \mathbb{R}^{2 n \times 2 n}$ and $\mathbf{S}_{z z} \in \mathbb{R}^{m \times m}$.

The matrix $\mathbf{L}=\mathbf{J S}$ is explicitly written as

$$
\mathbf{L}=\left(\begin{array}{cc}
\mathbf{J}_{0} & 0_{2 n, m} \\
0_{m, 2 n} & 0_{m, m}
\end{array}\right)\left(\begin{array}{cc}
\mathbf{S}_{x x} & \mathbf{S}_{x z} \\
\mathbf{S}_{z x} & \mathbf{S}_{z z}
\end{array}\right)=\left(\begin{array}{cc}
\mathbf{J}_{0} \mathbf{S}_{x x} & \mathbf{J}_{0} \mathbf{S}_{x z} \\
0_{m, 2 n} & 0_{m, m}
\end{array}\right)
$$

and clearly $\operatorname{rank}(\mathbf{L}) \leqslant 2 n$. Furthermore, $\operatorname{rank}\left(\mathbf{S}_{x x}\right)<2 n$ because $y_{0}$ is a critical stationary point at which the stability changes and we assumed that an eigenvalue passes the origin (steady-state bifurcation). The generic case gives $\operatorname{rank}\left(\mathbf{S}_{x x}\right)=$ $2 n-1$ together with $\operatorname{rank}(\mathbf{L})=2 n$ due to contribution from $\mathbf{J}_{0} \mathbf{S}_{x z}$. Under this setting we show the following claim which explains the generic existence of a size-3 Jordan block at bifurcating points in noncanonical Hamiltonian systems, as was observed on the Vlasov-HMF example in Sec. III B.

Claim. If $\mathbf{L}$ has a nontrivial 0 eigenvalue, then, generically, the associated generalized eigenspace is of dimension 3: if $\psi^{(0)} \in \mathbb{R}^{2 n} \times\left\{0_{m}\right\}$ is an eigenvector, i.e. $\mathbf{L} \psi^{(0)}=0_{2 n+m}$, there exist $\psi^{(1)}$ and $\psi^{(2)}$ such that

$$
\mathbf{L} \psi^{(1)}=\psi^{(0)}, \quad \mathbf{L} \psi^{(2)}=\psi^{(1)},
$$

and there is no solution $\psi^{(3)}$ to the equation $\mathbf{L} \psi^{(3)}=\psi^{(2)}$. $\mathbf{L}$ has the structure (42) when it is restricted to the subspace $\operatorname{Span}\left\{\psi^{(0)}, \psi^{(1)}, \psi^{(2)}\right\}$. Our proof stands for the following fact and two corollaries:

Fact. The linear equation $\mathbf{L} \psi=v$ has a solution $\psi$ if and only if $v$ is orthogonal to $\operatorname{Ker}^{\dagger}$, i.e., $\langle\varphi, v\rangle_{n, m}=0$ for any $\varphi \in \operatorname{Ker} \mathbf{L}^{\dagger}$; here $\langle\cdot, \cdot\rangle_{n, m}$ is the inner product on $\mathbb{R}^{2 n+m}$ and $\mathbf{L}^{\dagger}$ is the adjoint operator (transposition) of $\mathbf{L}$.

Corollary 1 (Cor 1). The equation $\mathbf{L} \psi=v$ has a solution if and only if the last $m$ coordinates of $v$ vanish.

Proof. $\operatorname{Ker} \mathbf{L}^{\dagger}=\left\{0_{2 n}\right\} \times \mathbb{R}^{m}$. Saying that $v$ is orthogonal to $\operatorname{Ker} \mathbf{L}^{\dagger}$ is equivalent to saying that the last $m$ coordinates of $v$ vanish.

Corollary 2 (Cor 2). The equation $\mathbf{J}_{0} \mathbf{S}_{x x} \xi=w$ has a solution if and only if $\left\langle\mathbf{J}_{0} \xi_{0}, w\right\rangle_{n}=0$, where $\xi_{0}$ is a vector spanning $\operatorname{Ker}\left(\mathbf{S}_{x x}\right)$ and $\langle\cdot, \cdot\rangle_{n}$ is the inner product on $\mathbb{R}^{2 n}$.

Proof. We show $\operatorname{Ker}\left(\mathbf{J}_{0} \mathbf{S}_{x x}\right)^{\dagger}=\operatorname{Span}\left\{\mathbf{J}_{0} \xi_{0}\right\}$. We first note that $\operatorname{dim} \operatorname{Ker}\left(\mathbf{S}_{x x}\right)=1$, hence $\operatorname{Ker}\left(\mathbf{S}_{x x}\right)$ is indeed spanned by a single vector $\xi_{0}$. We have $\left(\mathbf{J}_{0} \mathbf{S}_{x x}\right)^{\dagger}=-\mathbf{S}_{x x} \mathbf{J}_{0}$ and hence its kernel is spanned by $\mathbf{J}_{0} \xi_{0}$ by using $\mathbf{J}_{0}^{-1}=-\mathbf{J}_{0}$.

Proof of the claim. Let us write a vector $\psi$ on $\mathbb{R}^{2 n+m}$ as $\psi=(\xi, \zeta)^{\mathrm{T}}\left(\xi \in \mathbb{R}^{2 n}, \zeta \in \mathbb{R}^{m}\right)$, where $\mathrm{T}$ represents the transposition for vectors, but we will omit it from now on to simplify notations, since no confusion occurs. A critical eigenvector satisfying $\mathbf{L} \psi^{(0)}=0_{2 n+m}$ is $\psi^{(0)}=\left(\xi^{(0)}, 0_{m}\right)$. By
Cor 1 the equation $\mathbf{L} \psi^{(1)}=\psi^{(0)}$ has a solution. Writing $\psi^{(1)}=\left(\xi^{(1)}, \zeta^{(1)}\right)$, we have

$$
\mathbf{J}_{0} \mathbf{S}_{x x} \xi^{(1)}+\mathbf{J}_{0} \mathbf{S}_{x z} \zeta^{(1)}=\xi^{(0)}
$$

Since $\left\langle\mathbf{J}_{0} \xi^{(0)}, \xi^{(0)}\right\rangle_{n}=0$, by Cor 2 the above equation has a solution with $\zeta^{(1)}=0_{m}$, and we have found the first generalized eigenvector $\psi^{(1)}=\left(\xi^{(1)}, 0_{m}\right)$.

Again by Cor $1 \mathbf{L} \psi^{(2)}=\psi^{(1)}$ has a solution because the last $m$ coordinates of $\psi^{(1)}$ vanish. However, in general $\left\langle\mathbf{J}_{0} \xi^{(0)}, \xi^{(1)}\right\rangle_{n} \neq 0$, hence by Cor 2 it is impossible to find $\psi^{(2)}$ with a vanishing second component: the solution is of the form $\psi^{(2)}=\left(\xi^{(2)}, \zeta^{(2)} \neq 0_{m}\right)$. It is now clear, by Cor 1 again, that $\mathbf{L} \psi=\psi^{(2)}$ has no solution, and there are only two generalized eigenvectors, forming a size-3 Jordan block. Furthermore, $\psi^{(2)}$ has a nonzero component $\zeta^{(2)}$ along the direction of the Casimir invariants.

Remark 5. This coupling with the Casimir invariants is the specificity of this bifurcation. Indeed if the Hamiltonian does not induce any coupling with the Casimir modes at the linear level, i.e., $\mathbf{S}_{x z}=0_{2 n, m}$, the assumption $\operatorname{rank}(\mathbf{L})=2 n$ breaks down, and $\operatorname{rank}(\mathbf{L})=2 n-1$ instead. Generically $\mathbf{L}$ then has a size-2 Jordan block, without coupling with the Casimir modes, because $\operatorname{KerL}^{\dagger}$ has one more dimension. This size-2 Jordan block would also be the generic case for a generic nondegenerate finite dimensional Hamiltonian. A simple example is $H=p^{2} / 2-\mu q^{2} / 2$, whose equation of motion has a size-2 Jordan block at the critical point $\mu=0$ :

$$
\frac{d}{d t}\left(\begin{array}{l}
q \\
p
\end{array}\right)=\left(\begin{array}{ll}
0 & 1 \\
\mu & 0
\end{array}\right)\left(\begin{array}{l}
q \\
p
\end{array}\right)
$$

\section{B. Nonlinear study: Normal form}

Now that the structure of the linearized Vlasov operator at the bifurcating point is established, this section is devoted to the derivation of an effective dynamics in reduced dimension, for the evolution of an initial condition which is a perturbation of a weakly unstable stationary state. Our approach is to construct a normal form on the reduced space, instead of computing the explicit projection in (61) because the normal form must include the projected dynamics. We show that the normal form we obtain is essentially the same as the reduced noncanonical Hamiltonian system (60) of the Vlasov-HMF model. This approach shows that the result of the VlasovHMF case is not related to the simplicity of the system and is more general. On the other hand, such a formal approach does not yield explicit expressions for the parameters of the reduced dynamics, which precludes a direct quantitative comparison with numerics. In Sec. IV C we provide a qualitative analysis of the normal form.

We start from the noncanonical Hamiltonian system

$$
\dot{u}=\mathbf{J}_{\text {red }} \nabla H(u, \mu),
$$

where $u=\left(u_{0}, u_{1}, u_{2}\right), \mathbf{J}_{\text {red }}$ is a degenerate Poisson matrix

$$
\mathbf{J}_{\text {red }}=\left(\begin{array}{ccc}
0 & 1 & 0 \\
-1 & 0 & 0 \\
0 & 0 & 0
\end{array}\right)
$$

and the Hamiltonian $H$ is formally expanded as

$$
H(u, \mu)=H_{2}(u, \mu)+H_{3}(u),
$$


where $H_{k}$ are homogeneous polynomials of degree $k$ in its $u$ variables. The parameter $\mu$ controls the stability of the origin and we keep only the leading terms in $\mu$, of order $\mu u^{2}$.

The idea of the normal form is to make the Hamiltonian $H$ as simple as possible through changes of variables. We first set the quadratic part $\mathrm{H}_{2}$ as

$$
H_{2}(u, \mu)=\left(u_{1}^{2}-\mu u_{0}^{2}\right) / 2-u_{0} u_{2},
$$

so that the linear dynamics recovers the size-3 Jordan block and the change of stability of the origin at $\mu=0$ :

$$
\mathbf{J}_{\mathrm{red}} \nabla H_{2}=\left(\begin{array}{c}
u_{1} \\
\mu u_{0}+u_{2} \\
0
\end{array}\right)=\left(\begin{array}{ccc}
0 & 1 & 0 \\
\mu & 0 & 1 \\
0 & 0 & 0
\end{array}\right)\left(\begin{array}{l}
u_{0} \\
u_{1} \\
u_{2}
\end{array}\right) .
$$

We transform the coordinates from $u$ to $U=(Q, P, Z)$ defined as $u=U+T(U)$, and choose $T$ in order to simplify $\bar{H}(U):=H(U+T(U))$. We assume that each component of the three-dimensional vector $T(U)$ is a homogeneous polynomial of order 2 and thus has $3 \times(3 \times 2)=18$ parameters. Imposing to keep the standard form (68) for $\mathbf{J}_{\text {red }}$ reduces the number of free parameters to 10 . The cubic term of $\bar{H}(U)$ is $H_{3}(U)+T \cdot \nabla H_{2}(U)$. All terms in $H_{3}(U)$ can be eliminated by appropriate choices of the 10 free parameters left in $T$, except the $Q^{3}$ and the $Z Q^{2}$ ones. Finally, the coefficient of the remaining $Q^{3}$ term can be scaled to 1 , and, since $Z$ is conserved by the dynamics, the $Z Q^{2}$ term can be absorbed in a redefinition of $\mu$ : the details of how this normal form is obtained are given in Appendix C. Consequently, around the critical point, the normal form of the reduced Hamiltonian is

$$
H_{\text {red }}=P^{2} / 2+\Phi(Q, Z), \quad \Phi=-\mu Q^{2} / 2-Q Z+Q^{3}
$$

up to cubic order, and the equations of motion are

$$
\begin{aligned}
& \dot{Q}=P \\
& \dot{P}=Z-3 Q^{2}+\mu Q . \\
& \dot{Z}=0
\end{aligned}
$$

Up to simple scaling, we have indeed found the same Poisson structure and essentially the same Hamiltonian as in the Vlasov-HMF case; see (60).

\section{Qualitative study of the normal form}

Figure 1 represents the phase space portraits of Hamiltonian (72), depending on the values of $\mu$ and $Z$. It shows a kind of three-dimensional "fish"-shape bifurcation [8], with an important observation: the value of the Casimir invariant, $Z$, appears as a second parameter controlling the bifurcation. Lie-Darboux theorem tells that Poisson manifold can be decomposed as stacks of symplectic manifold parametrized by the value of Casimir invariants [11]. This structure is explicitly observed in our reduced system with the three-dimensional Jordan block. Figure 1 provides an illustration where $Z$ is the Casimir parameter.

Stationary states of the system (73) are, if $\mu^{2}+12 Z \geqslant 0$, $(Q, P)=\left(\mu \pm \sqrt{\mu^{2}+12 Z}, 0\right)$, from which we emphasize the following points:

(1) When the reference stationary state is unstable $(\mu>0)$, there are two regimes: when $\mu^{2}+12 Z<0$, there is no stationary point, and all trajectories diverge; when $\mu^{2}+12 Z>0$,

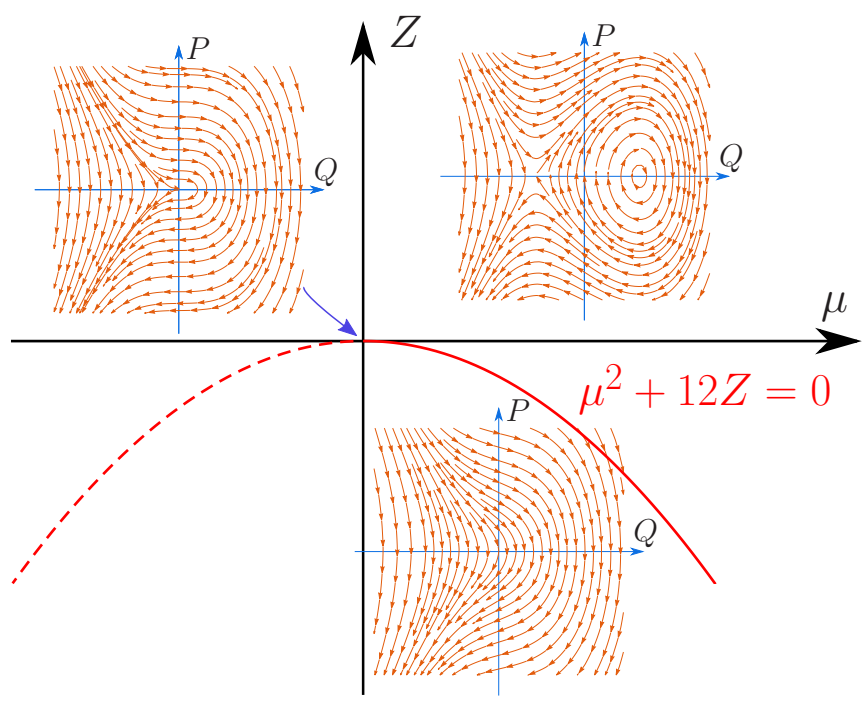

FIG. 1. Sketch of the phase space portraits according to the reduced Hamiltonian (72). In the region $\mu>0$, the dynamics strongly depends on $Z$, which is fixed by the initial perturbation. The equation of the boundary (red curve) is $\mu^{2}+12 Z=0$. In the region $\mu<0$, $(Q, P, Z)=(0,0,0)$ is a stable stationary state. However, because of the infinite dimensional nature of the Vlasov equation and at variance with the finite dimensional cases, there are not necessarily purely imaginary eigenvalues close to 0 (as they may collide with the continuous spectrum), and the reduced Hamiltonian may not be meaningful for $\mu<0$.

there are two stationary states, one saddle point $(Q, P)=$ $\left(\mu-\sqrt{\mu^{2}+12 Z}, 0\right)$ and one stable $\left(\mu+\sqrt{\mu^{2}+12 Z}, 0\right)$.

(2) Hence, when $\mu^{2}+12 Z>0$, the fate of the dynamics can be very different, depending on the initial perturbation: either small stable oscillations close to the reference stationary state, or a diverging trajectory.

(3) The oscillation's amplitude is determined by the position of the stable stationary state, $\mu+\sqrt{\mu^{2}+12 Z}$. If $Z=0$, the amplitude scales as $\mu$, which is of order $\lambda^{2}$, where $\lambda$ is the instability rate [as can be easily checked from (71)], but even a small $Z$ modifies this scaling: if $Z \gg \lambda^{4}$, the amplitude scales as $\sqrt{Z}$.

These findings generalize those obtained in Ref. [29] using an unstable manifold expansion. Validity of Fig. 1 in a Vlasov system will be examined in Sec. V.

\section{EXPLICIT REDUCTION AND NUMERICAL SIMULATIONS FOR THE VLASOV-HMF EXAMPLE}

We come back now to explicit computations for the Vlasov-HMF case in order to demonstrate that the bifurcation represented in Fig. 1 indeed occurs and accurately describes, in a quantitative manner, the dynamical behavior of the system in the vicinity of the bifurcation point.

\section{A. A bifurcating family of stationary states}

We take the family of "Fermi-Dirac" stationary states

$$
F_{\mu}(J)=\mathcal{N}^{-1} \frac{1}{1+e^{\beta[H(J)-(\mu-\kappa)]}},
$$


where $\mathcal{N}^{-1}$ is the normalization factor, $\mu$ controls the bifurcation, and $\kappa$ is chosen so that the critical point is $\mu=$ 0 (we fix $\beta=40$ and $\kappa=0.6693$ accordingly): for $\mu<0$, the spectrum of the linearized dynamics around $F_{\mu}$ is purely imaginary, and small perturbations damp away due to a kind of Landau damping [34]. A real positive eigenvalue appears for $\mu>0$ [29], indicating that $F_{\mu}$ becomes unstable. The critical stationary state at $\mu=0$ has magnetization $M_{0}=$ 0.3361 . We then perturb a slightly unstable stationary state with $\mu=1.44 \times 10^{-4}, M_{\mu}=0.3360$ (it has an associated unstable eigenvalue $\lambda=8.62 \times 10^{-3}$ ) as

$$
F(t=0)-F_{\mu}=F_{T}=\varepsilon \cos q \times e^{-\beta_{T} p^{2}},
$$

with a fixed $\beta_{T}=10$ and different perturbation amplitudes $\varepsilon$.

Following Sec. III C, we can reduce the evolution of the perturbation to the Hamiltonian dynamics (59), with explicit expressions of the changes of variables, coefficients, and initial conditions involved. In particular, we have computed the coefficient in (59) associated with the linear term of $O_{1}\left(\mu, M_{\mu}\right): \mu a \simeq 7.44 \times 10^{-5}$, as well as the ones involved in the quadratic terms $C_{010}=-0.409, C_{100} \simeq-0.181$. The initial conditions are found to be $Q(0) \simeq 1.42 \varepsilon, P(0)=0$, $Z(0) \simeq 0.445 \varepsilon$. We see that changing $\varepsilon$ simultaneously affects $Q(0)$ and $Z(0)$. Note that all the coefficients and the initial condition are explicitly computed with their derived expression and thus we directly compare without any fitting parameters the direct numerical simulations (DNS) and the reduced dynamics $(3 \mathrm{D})$.

The quantity $Q(t)$ in the reduced dynamics is, at the leading order, the projection of the perturbation on the eigenvector $\psi^{(0)}$; recalling that the magnetization $M\left[\psi^{(0)}\right]=1$, whereas $M\left[\psi^{(1)}\right]=M\left[\psi^{(2)}\right]=0$, we conclude that at the leading order $Q(t)$ is proportional to the magnetization of the perturbation, i.e., $M[F(t)]-M_{\mu}$. This is important because the magnetization is the main physical characteristic of the system; it is also numerically very easy to compute. We must keep in mind however that the relation between $Q$ and the magnetization is valid only for small amplitudes of perturbations.

\section{B. Comparison between direct numerical simulations and the reduced dynamics}

Following Sec. V A, we plot the time evolution of the magnetization $M[F(t)]-M_{\mu}$ (see details in Sec. V C) and compare it to $Q(t)$ obtained from the analytic solution (B4) to the reduced model (60).

The comparison in Fig. 2 prompts several remarks:

(1) The agreement is good, in terms of both frequency and amplitude, over fairly long timescales, especially keeping in mind that there is no adjustable parameter.

(2) There is a small damping (and frequency shift) acting on DNS, an effect that we attribute to the numerical dissipation well known in Vlasov simulations [36,37]. We confirm this hypothesis in Sec. V C by testing different grid sizes. However, one cannot exclude the possibility of a weak Landau damping-like effect not described by the reduced Hamiltonian.

(3) Changing the initial perturbation amplitude has an important effect on the dynamics: this is a signature of the

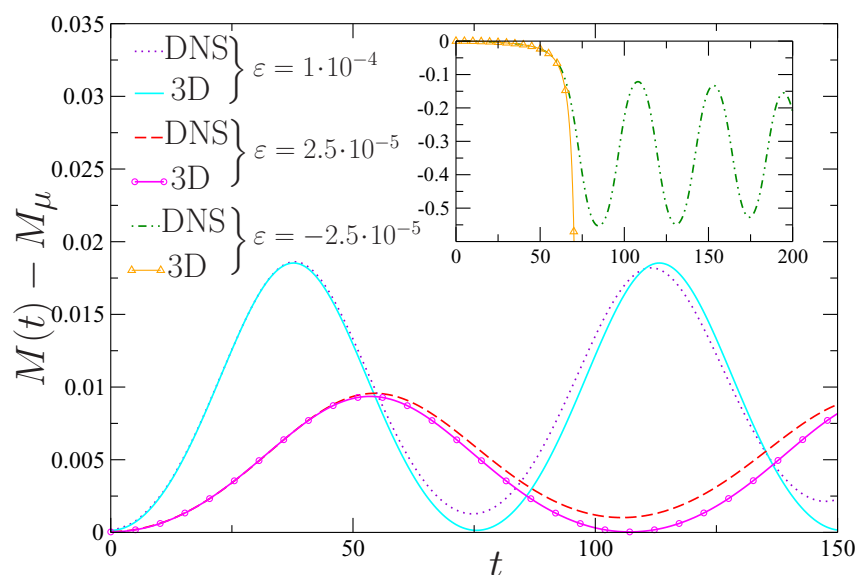

FIG. 2. Temporal evolution of magnetization. Comparison between the Vlasov-HMF model (25) (DNS, dashed and dotted lines) and the reduced model (60) (3D, solid lines). Two different amplitudes for the initial perturbation are used. In the inset we show DNS and three dimensions for the same initial state with $\varepsilon<0$ (resulting in $\mu^{2}+12 Z<0$ ); the three-dimensional dynamics diverges, while the DNS shows large-scale oscillations. See the discussion for details of the numerical simulations.

importance of the $Z$ coordinate, representing the coupling with the Casimir invariants.

(4) The Vlasov dynamics indeed leaves the perturbative regime as predicted by the reduced model and the magnetization does not come back to the initial level (see inset and the end of Appendix C). The observed large-scale oscillations suggest the existence of a periodic solution; they are out of reach of the reduced model, but it is worth mentioning that a reduced Hamiltonian at fourth order indeed suggests in some cases the confinement of trajectories and large-scale oscillations.

\section{Details on the numerical method and potential issues}

The Vlasov-HMF model (25) is simulated with the algorithm of Ref. [35], based on a second order time-split algorithm with a local modified cubic-spline interpolation. In our simulations the phase space $(q, p)$ is divided into $N_{q} \times N_{p}$ grid elements with $N_{q}=N_{p}=4096$. We have $q \in[-\pi, \pi]$ and cut off the velocity region to $p_{\max }=-p_{\min }=2$ (which is enough to well capture the whole $F$ density). It corresponds to $\Delta q=2 \pi / N_{q} \simeq 1.5 \times 10^{-3}$ and $\Delta p=2 p_{\max } / N_{p} \simeq 9.8 \times$ $10^{-4}$. The time step is $\Delta t=5 \times 10^{-3}$.

In the following we identify two challenges for the comparison between DNS and the three-dimensional reduced dynamics:

(1) Numerical error of DNS. The choices of the grid elements number $N_{q}, N_{p}$, maximum velocity $p_{\max }$ and time step $\Delta t$ are crucial. Indeed, a large number of grid elements is needed to resolve the fine scales in the region of interest. In that sense a small $p_{\max }$ is good but at the same time $p_{\max }$ has to be large enough to capture the tails of the density $F(q, p, t)$ which might affect the dynamics. In addition, practically, all the numerical parameters must respect some stability condition. To assess different 


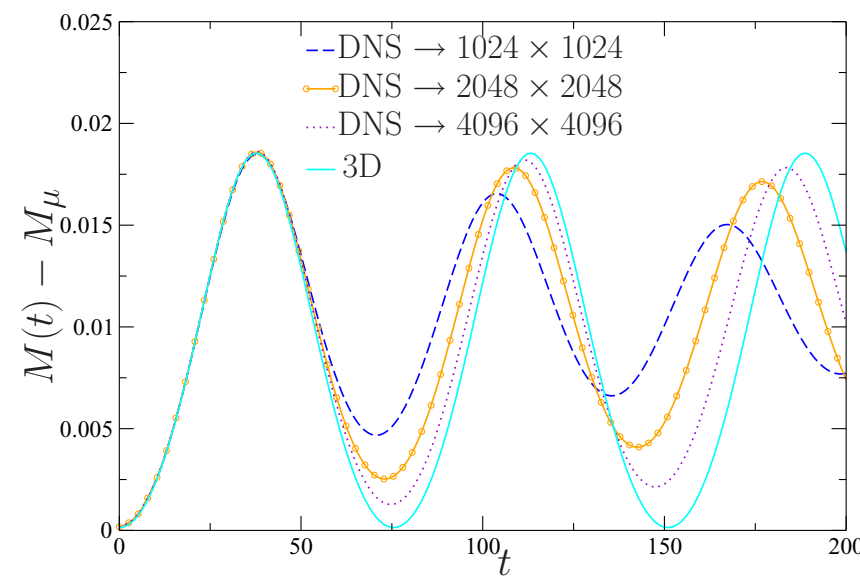

FIG. 3. Magnetization as a function of time: comparison at $\varepsilon=$ $1 \times 10^{-4}$ between direct numerical simulations (DNS) of Eq. (25) for different phase space grid number $N_{q} \times N_{p}$ and the analytic solution to three dimensions, Eq. (60).

sets of parameters, we compute the relative errors in energy conservation $\operatorname{err}_{\mathcal{E}}=[\mathcal{E}(\mathrm{t})-\mathcal{E}(0)] / \mathcal{E}(0)$ and norm conservation $\operatorname{err}_{\mathcal{N}}=[\mathcal{N}(\mathrm{t})-\mathcal{N}(0)] / \mathcal{N}(0)$ : total energy $\mathcal{E}(t)$ and total norm $\mathcal{N}(t)$ are exactly conserved by the Vlasov equation. Typically at $t=200$ we achieve $\operatorname{err}_{\mathcal{N}} \sim 10^{-13}$ and $\operatorname{err}_{\mathcal{E}} \sim 10^{-9}$.

We have also verified that, all other parameters remaining constant, decreasing the number of grid elements increases the numerical damping or shifting caused by the numerical solver (this is well known in Vlasov simulations [36]). We illustrate the phenomenon in Fig. 3, where we clearly see that higher $N_{q}$ and $N_{p}$ diminish the effect of numerical damping and shifting. The DNS seems to converge toward the reduced model when the grid numbers $N_{q}$ and $N_{p}$ increase.

(2) Initial conditions. The reduced dynamics is a priori valid for an initial condition on the invariant manifold $\mathcal{M}_{0}$, that is approximately on the subspace $E_{0}$. However, the vectors $\psi^{(i)}$ have a singularity at the separatrix [from the functions $C_{\alpha}(J)$ ] which entails large discretization errors at the grid level. Rather, we have used a smooth perturbation $F_{T}$, as in Eq. (75), which is not in $E_{0}$. The initial condition for three dimensions $(Q(0), P(0), Z(0))$ is then explicitly computed by the following scalar products $A_{0}(t=0)=$ $\left\langle\phi^{(2)}, F_{T}\right\rangle, A_{1}(t=0)=\left\langle\phi^{(1)}, F_{T}\right\rangle=0$ (by symmetry of $F_{T}$ ) and $A_{2}(t=0)=\left\langle\phi^{(0)}, F_{T}\right\rangle$. The good agreement seen in Fig. 2 suggests that the component of $F_{T}$ which is not in $E_{0}$ has a small effect on the dynamics, and that the reduced dynamics is relevant for more general initial conditions than those on $\mathcal{M}_{0}$.

\section{PHYSICAL EXAMPLES AND DISCUSSION}

To summarize, we have identified and described on general theoretical grounds a new type of bifurcation for Vlasov systems, and then proved that it indeed occurs on a simple system. We discuss now its possible relevance in more realistic physical systems: we need to find situations where the basic conditions of the weak resonance and possible coupling with Casimir modes are satisfied.
Instabilities of Bernstein-Greene-Kruskal (BGK) modes in plasmas provide a vast class of natural candidates, since they are nonhomogeneous steady states (in some reference frame) of the Vlasov equation. The simplest cases, based on the one-dimensional Vlasov-Poisson equation, are similar to the HMF example studied above, and we know that bifurcations do occur (see, for instance, Ref. [38]): we expect that when a BGK mode becomes unstable, the behavior of the instability may, at least in some instance, be described by the theory put forward in this paper.

Radial orbit instability is well known in astrophysics (see, for instance, Ref. [39]), and believed to play a role in determining the structure of galaxies. It occurs in self-gravitating systems, when the amount of particles (usually stars) with small angular momentum increases. The nonlinear analysis in Ref. [40] suggests similarities with the phenomenology of Fig. 1: in particular, the instability is nonoscillating, and, depending on the initial perturbation, the saturated state may be close to the reference stationary state, or far away. Still in astrophysics, gravitational loss cone instability (see, for instance, Ref. [41]) could also present a similar phenomenology, however we are not aware of a nonlinear analysis of this situation.

A class of Hamiltonian models of oscillators synchronization is introduced in Ref. [42]: identical nonlinear oscillators are coupled through a mean-field, and the stationary state with all oscillators desynchronized can undergo a bifurcation towards a synchronized state. We believe this bifurcation should be in some cases similar to the one described in this article, with the caveat that no coupling with Casimir invariants takes place; accordingly, the Jordan block at criticality has only size two. This type of models can for instance describe coupled electric circuits [42], as well as, somewhat unexpectedly, pressure waves in bubbly fluids $[43,44]$.

We conclude that many physical systems from very different fields can be expected to follow the phenomenology in Fig. 1, as the reduced model was formally obtained for a generic class of noncanonical Hamiltonian systems; specific studies and simulations in each case are now needed to confirm or infirm these predictions, and assess their physical importance.

\section{ACKNOWLEDGMENTS}

D.M. carried out the work at LANL under the auspices of the National Nuclear Security Administration of the U.S. Department of Energy under Contract No. DE-AC52-06NA25396 and was partially supported by LANL/LDRD/CNLS projects. Y.Y.Y. acknowledges the support of JSPS KAKENHI Grant No. 16K05472.

\section{APPENDIX A: THREE-DIMENSIONAL JORDAN BLOCK STRUCTURE IN ONE-DIMENSIONAL VLASOV EQUATIONS}

This Appendix provides the details of the computations corresponding to Sec. III B, in the case of a general two-body potential $v(q)$. The Vlasov-HMF case can be easily recovered from what follows. 
We consider a generic one-dimensional Vlasov system

$$
\begin{aligned}
& \frac{\partial F}{\partial t}+p \frac{\partial F}{\partial q}-\frac{\partial V[F]}{\partial q} \frac{\partial F}{\partial p}=0, \\
& \quad \text { with } V[F](q, t)=\iint v\left(q-q^{\prime}\right) F\left(q^{\prime}, p^{\prime}, t\right) d q^{\prime} d p^{\prime},
\end{aligned}
$$

where $q \in[0,2 \pi), p \in \mathbb{R}$ and $v(q)$ is the two-body interaction potential. Thanks to the periodic boundary condition, spatial Fourier series are a natural expansion for the interaction potential $v$ and the density $F$; in a setting where $q$ is unbounded, one would have to use other expansions. The two-body potential is expanded as

$$
v(q)=\sum_{k \in \mathbb{Z}} v_{k} e^{i k q}
$$

and must be even from the law of action and reaction. We can take $v_{0}=0$ without loss of generality. The Vlasov-HMF case of Sec. III B corresponds to $v_{1}=v_{-1}=-1 / 2$, and $v_{k}=0$ if $k \neq \pm 1$. We will show that the linearized Vlasov equation around a stationary state has a size-3 Jordan block structure (41), and give the expressions of the generalized eigenvectors and eigenprojections at the critical point [Eqs. (36)-(38) and (45) for the HMF case]. Note that, in this Appendix, we will omit the upper tilde on Fourier components for simplicity of notation, while the subscript 0 of $F_{0}$ represents the critical point $\mu=0$. We also omit the subscript 0 of the linear operators $\mathcal{L}_{0}$ and $\mathcal{L}_{0}^{\dagger}$ because we consider solely the critical point.

\section{Symmetry of Fourier components}

As mentioned in Sec. III B, a stationary state $F_{0}$ gives an integrable Hamiltonian $h$ and they are functions of the action variable $J$. A function $g$ defined on the phase space $(q, p)$ is hence expanded into the Fourier series of the angle variable $\theta$ as

$$
g(\theta, J)=\sum_{\alpha \in \mathbb{Z}} g_{\alpha}(J) e^{i \alpha \theta} .
$$

We will need to switch between position-momentum and angle-action coordinates; we introduce for this purpose the functions

$$
c_{k, \alpha}(J)=\frac{1}{2 \pi} \int_{0}^{2 \pi} e^{i k q(\theta, J)} e^{-i \alpha \theta} d \theta .
$$

Before entering into details of the computations, we give two remarks.

The first remark is on the $(\theta, J)$ notation. The phase space may be divided into several parts in each of which the angleaction variables are defined separately. For instance, in the case $v(q)=-\cos q$ and when the mean-field potential does not vanish, the phase space has a separatrix and is divided into three parts, as seen in Fig. 4 (see Ref. [34] for details): inside the separatrix, the upper side of separatrix, and the lower side of separatrix. The above expressions such as (A2) or (A3) then use the notation $(\theta, J)$ as a convenient short-hand for this more complicated structure. To give a precise example, in a one-dimensional Hamiltonian system, we have to understand the definition (A3) of the function $c_{k, \alpha}$ as follows: a periodic

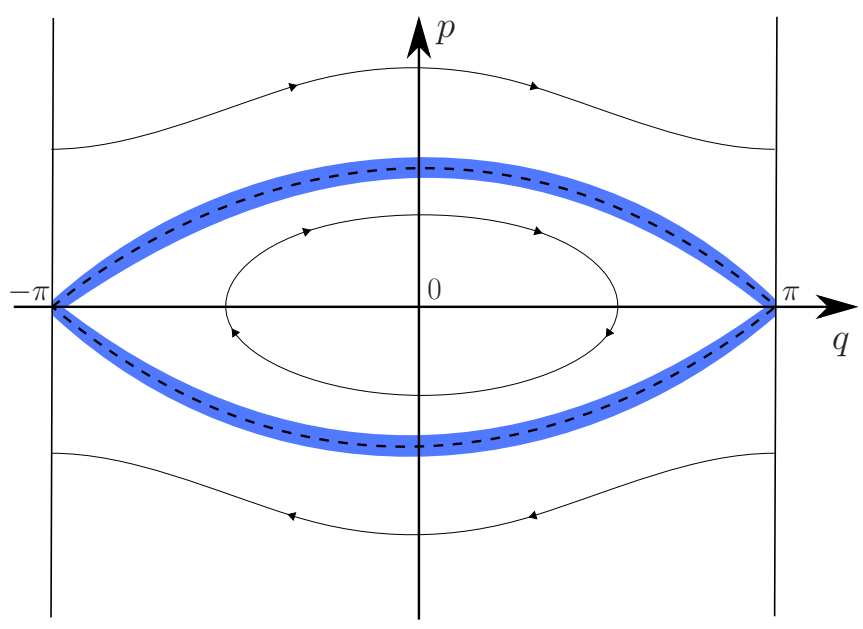

FIG. 4. A schematic picture of the phase space in the HMF model. Each energy contour (a curve) is identified by the action variable $J$, and the arrows show the direction in which the angle variable $\theta$ increases along the curve. The shaded area is a weak-resonance region with the critical zero frequency.

orbit $\varphi$ corresponds to an iso- $J$ line, and $c_{k, \alpha}$ is actually a function of this orbit

$$
c_{k, \alpha}(\varphi)=\frac{\Omega_{0}(\varphi)}{2 \pi} \int_{0}^{T(\varphi)} e^{i k \varphi_{q}(t)} e^{-i \alpha \Omega_{0}(\varphi) t} d t,
$$

where $T(\varphi)$ and $\Omega_{0}(\varphi)$ are the period and the frequency of the orbit $\varphi=\left(\varphi_{q}, \varphi_{p}\right), \varphi_{q}(t), \varphi_{p}(t)$ are the position and momentum coordinates along the orbit $\varphi$ and we assumed $\Omega_{0}(\varphi) \neq 0$.

The second remark is on the symmetry of $c_{k, \alpha}(J)$, which is revealed by considering the time-reversed orbit $R[\varphi](t)=$ $\left(\varphi_{q}(-t),-\varphi_{p}(-t)\right)$. The time-reversed orbit exists due to the symmetry $h(q,-p)=h(q, p)$. Between the two orbits $\varphi$ and $R[\varphi]$, we have the symmetry

$$
c_{k, \alpha}(R[\varphi])=c_{k,-\alpha}(\varphi) .
$$

Later we will find integrations over the action $J$. This integration has to be understood as an integration over the orbits foliating the phase space. Changing the foliation from $\{\varphi\}$ to $\{R[\varphi]\}$, roughly speaking, the symmetry (A5) permits to replace $c_{k, \alpha}$ with $c_{k,-\alpha}$.

\section{Linear operator $\mathcal{L}$ and its generalized eigenvectors}

Let us denote the linear operator around the stationary state $F_{0}$ as $\mathcal{L}$, where we omitted the subscript 0 for a simple notation. The operator $\mathcal{L}$ is of the form (33), which is expanded into Fourier series as

$$
(\mathcal{L} \cdot f)_{\alpha}=-i \alpha \Omega_{0}(J) f_{\alpha}(J)+i \alpha F_{0}^{\prime} \sum_{k \in \mathbb{Z}} v_{k} Y_{k}[f] c_{k, \alpha}(J),
$$

where $(\mathcal{L} \cdot f)_{\alpha}$ represents the $\alpha$ th Fourier component of $\mathcal{L} \cdot f$ with respect to the angle variable $\theta$ and

$$
Y_{k}[f](J)=2 \pi \sum_{\alpha \in \mathbb{Z}} \int f_{\alpha}(J) c_{k, \alpha}^{*}(J) d J .
$$

We assume that $F_{0}$ is a critical stationary state: it has a bifurcating 0 eigenvalue. We look for the associated eigenvector 
$\psi^{(0)}$ which induces the generalized eigenvectors $\psi^{(n)}$ and makes a Jordan block as large as possible. To obtain a solution to $\mathcal{L} \cdot \psi^{(m+1)}=\psi^{(m)}$, for $\alpha=0, \psi_{\alpha=0}^{(m)}=0$ is necessary because we always have $\left(\mathcal{L} \cdot \psi^{(m+1)}\right)_{\alpha=0}=0$.

The first step is to find $\psi^{(0)}$, solving $\mathcal{L} \cdot \psi^{(0)}=0$. From (A6), we see that the equation for $\alpha=0$ is always satisfied; hence we may choose any function for $\psi_{\alpha=0}^{(0)}(J)$. As commented above, we take $\psi_{\alpha=0}^{(0)}(J)=0$ in order to be able to find a first generalized eigenvector later. Notice that this choice of $\psi_{0}^{(0)}$ corresponds to perturbations that do not modify the values of the Casimir invariants at linear order; see (49). For $\alpha \neq 0$, we have

$$
\psi_{\alpha}^{(0)}(J)=\frac{F_{0}^{\prime}(J)}{\Omega_{0}(J)} \sum_{l \in \mathbb{Z}} v_{l} Y_{l}\left[\psi^{(0)}\right] c_{l, \alpha}(J) .
$$

The weak resonance hypothesis ensures that the above expression is regular (or at least integrable): there are no or few particles with 0 frequency $\left(1 / \Omega_{0}\right.$ may have a logarithmic divergence; this indeed happens whenever there is a separatrix trajectory and may be called "weak resonance"). Both sides of (A8) contain $\psi^{(0)}$, and, therefore, $Y_{l}\left[\psi^{(0)}\right]$ must be determined self-consistently. Inserting (A8) in (A7) with the choice of $\psi_{0}^{(0)}=0$, we obtain

$$
\sum_{l \in \mathbb{Z}} \Lambda_{k l} Y_{l}\left[\psi^{(0)}\right]=0
$$

with

$$
\Lambda_{k l}=\delta_{k l}-2 \pi v_{l} \sum_{\alpha \in \mathbb{R}} \int \frac{F_{0}^{\prime}(J)}{\Omega_{0}(J)} c_{k, \alpha}^{*}(J) c_{l, \alpha}(J) d J .
$$

The condition to obtain a nontrivial solution to (A9) is $\operatorname{Ker}(\Lambda)$ nontrivial, which is consistent with the criticality assumption of $F_{0}$. The generic case is that $\operatorname{Ker}(\Lambda)$ is of dimension 1 , which we now assume. We call $\left(y_{k}\right)$ an element of this kernel. Then we have for any $\alpha \neq 0$ :

$$
\psi_{\alpha}^{(0)}(J)=\frac{F_{0}^{\prime}(J)}{\Omega_{0}(J)} \sum_{l \in \mathbb{Z}} v_{l} y_{l} c_{l, \alpha}(J)
$$

We now look for a generalized eigenvector $\psi^{(1)}$, solving $\mathcal{L} \cdot \psi^{(1)}=\psi^{(0)}$. From (A6), we see that the equation for $\alpha=$ 0 is again always satisfied due to the choice $\psi_{\alpha=0}^{(0)}=0$, and $\psi_{\alpha=0}^{(1)}$ is free again. We choose again $\psi_{\alpha=0}^{(1)}=0$ to search a second generalized eigenvector. For $\alpha \neq 0$, we have

$$
\psi_{\alpha}^{(1)}(J)=\frac{F_{0}^{\prime}}{\Omega_{0}} \sum_{l \in \mathbb{Z}} v_{l} Y_{l}\left[\psi^{(1)}\right] c_{l, \alpha}-\frac{F_{0}^{\prime}}{i \alpha \Omega_{0}^{2}} \sum_{l \in \mathbb{Z}} v_{l} y_{l} c_{l, \alpha} .
$$

Inserting (A12) into (A7), we obtain

$$
\sum_{l \in \mathbb{Z}} \Lambda_{k l} Y_{l}\left[\psi^{(1)}\right]=-2 \pi \sum_{l \in \mathbb{Z}} v_{l} y_{l} \sum_{\alpha \neq 0} \int \frac{F_{0}^{\prime}}{i \alpha \Omega_{0}^{2}} c_{k, \alpha}^{*} c_{l, \alpha} d J .
$$

Let us remember the remark after (A5). In the right-hand side, changing the orbit $\varphi$ to $R[\varphi]$ does not change $F_{0}^{\prime}(J) /\left(i \alpha \Omega_{0}^{2}\right)$ but $c_{k, \alpha}^{*} c_{l, \alpha}$ becomes $c_{k,-\alpha}^{*} c_{l,-\alpha}$. This implies that the sum over $\alpha \neq 0$, in the right-hand side of (A13), vanishes for any $l \in \mathbb{Z}$. A solution $Y_{l}\left[\psi^{(1)}\right]$ must be, therefore, chosen from $\operatorname{Ker}(\Lambda)$ and the first term of (A12) is proportional to $\psi^{(0)}$. Therefore, we may choose $Y_{l}\left[\psi^{(1)}\right] \equiv 0$ and, for $\alpha \neq 0$,

$$
\psi_{\alpha}^{(1)}(J)=\frac{F_{0}^{\prime}(J)}{-i \alpha \Omega_{0}(J)^{2}} \sum_{l \in \mathbb{Z}} v_{l} y_{l} c_{l, \alpha}(J) .
$$

We now look for a further generalized eigenvector $\psi^{(2)}$, solving $\mathcal{L} \cdot \psi^{(2)}=\psi^{(1)}$. From (A6), we see that the equation for $\alpha=0$ is again always satisfied, and $\psi_{\alpha=0}^{(2)}$ is free again. We will choose $\psi_{\alpha=0}^{(2)}$ later. For $\alpha \neq 0$, we have

$$
\psi_{\alpha}^{(2)}(J)=\frac{F_{0}^{\prime}}{\Omega_{0}} \sum_{l \in \mathbb{Z}} v_{l} Y_{l}\left[\psi^{(2)}\right] c_{l, \alpha}+\frac{F_{0}^{\prime}}{(-i \alpha)^{2} \Omega_{0}^{3}} \sum_{l \in \mathbb{Z}} v_{l} y_{l} c_{l, \alpha} .
$$

The self-consistent equation for $Y_{l}\left[\psi^{(2)}\right]$ is

$$
\begin{aligned}
\sum_{l \in \mathbb{Z}} \Lambda_{k l} Y_{l}\left[\psi^{(2)}\right]= & 2 \pi \sum_{l \in \mathbb{Z}} v_{l} y_{l} \sum_{\alpha \neq 0} \int \frac{F_{0}^{\prime}}{(i \alpha)^{2} \Omega_{0}^{3}} c_{k, \alpha}^{*} c_{l, \alpha} d J \\
& +2 \pi \int \psi_{0}^{(2)} c_{k, 0}^{*} d J
\end{aligned}
$$

If we choose $\psi_{0}^{(2)}=0$, the right-hand side of (A16) is nonzero and the linear equation (A16) does not have a solution in general. However, it is possible to choose appropriately the function $\psi_{0}^{(2)}(J)$ in order to ensure that the sum in the righthand side vanishes, so that a solution exists; we can then take $\forall k, Y_{k}\left[\psi^{(2)}\right]=0$. This requires only that the $c_{k, 0}$ form a free family of functions. We have thus built a second generalized eigenvector for the nontrivial eigenvalue 0 .

We can now summarize:

$$
\begin{aligned}
\psi^{(0)} & =\left(\begin{array}{c}
0 \\
\frac{F_{0}^{\prime}(J)}{\Omega_{0}(J)} \sum_{l \in \mathbb{Z}} v_{l} y_{l} c_{l, \alpha}(J)
\end{array}\right), \\
\psi^{(1)} & =\left(\begin{array}{c}
0 \\
\frac{F_{0}^{\prime}(J)}{-i \alpha \Omega_{0}^{2}(J)} \sum_{l \in \mathbb{Z}} v_{l} y_{l} c_{l, \alpha}(J)
\end{array}\right), \\
\psi^{(2)} & =\left(\begin{array}{c}
F_{0}^{\prime(J)} \\
\psi_{0}^{(2)}(J) \\
\frac{F_{0}^{2} \Omega_{0}^{3}(J)}{(i \alpha)^{2}} v_{l} y_{l} c_{l, \alpha}(J)
\end{array}\right),
\end{aligned}
$$

where, in each eigenvector, the upper line represents the element for $\alpha=0$, and the lower one contains the expression for $\alpha \neq 0$. Crucially, $\psi_{0}^{(2)} \neq 0$, which has two consequences:

(1) The equation $\mathcal{L} \cdot \psi^{(3)}=\psi^{(2)}$ has no solution, hence the characteristic space is only of dimension 3 .

(2) $\psi^{(2)}$ has a nonzero component in the direction that modifies the values of the Casimir invariants, $\alpha=0$.

At the bifurcation point, the linearized operator $\mathcal{L}$ restricted to the subspace $\operatorname{Span}\left\{\psi^{(0)}, \psi^{(1)}, \psi^{(2)}\right\}$ is represented by the three-dimensional Jordan block (42) as found in the main text on the basis of genericity arguments.

Notice the general solution of (A6) yields an infinite number of other eigenvectors with eigenvalue 0 , with nonvanishing zeroth Fourier component, and thus not associated to a Jordan block:

$$
\Psi=\left(\begin{array}{c}
\Psi_{0}(J) \\
\frac{F_{0}^{\prime}(J)}{\Omega_{0}(J)} \sum_{l \in \mathbb{Z}} v_{l} z_{l} c_{l, \alpha}(J)
\end{array}\right)
$$

with

$$
(\Lambda z)_{l}=2 \pi \int \Psi_{0}(J) c_{l 0}^{*}(J) d J .
$$


Since $\Lambda$ is not invertible, this last equation imposes a condition on $\Psi_{0}(J)$. Clearly, it is possible to add to $\psi^{(2)}$ any eigenvector of the type (A20) while keeping the Jordan block structure. In particular, as already noticed in Sec. III B, this means that there is some freedom in the determination of $E_{0}=\operatorname{Span}\left\{\psi^{(0)}, \psi^{(1)}, \psi^{(2)}\right\}$, which is very good news: any perturbation which is in $\operatorname{Span}\left\{\psi^{(0)}, \psi^{(1)}, \psi^{(2)},(\Psi)\right\}$ [where $(\Psi)$ represents all eigenvectors of type (A20)] is actually in some $E_{0}$ subspace, at the cost of redefining $\psi^{(2)}$. This may be part of the reason why the reduced dynamics is relevant for more initial conditions than one may a priori think.

\section{Adjoint operator $\mathcal{L}^{\dagger}$ and its generalized eigenvectors}

The adjoint linear operator of $\mathcal{L}$ with respect to the standard inner product (21) is of the form (43) and is expanded into the Fourier series with respect to the angle variable $\theta$ as

$$
\begin{aligned}
\left(\mathcal{L}^{\dagger} \cdot g\right)_{\alpha}= & i \alpha \Omega_{0}(J) g_{\alpha}(J) \\
& -\sum_{k \in \mathbb{Z}} v_{k} c_{k, \alpha}(J) \sum_{\beta \in \mathbb{Z}} i \beta \int g_{\beta}\left(J^{\prime}\right) F_{0}^{\prime}\left(J^{\prime}\right) c_{k, \beta}^{*}\left(J^{\prime}\right) d J^{\prime} .
\end{aligned}
$$

We first look for $\phi^{(0)}$ such that $\mathcal{L}^{\dagger} \cdot \phi^{(0)}=0$. For $\alpha=0$, this imposes that for all $k$ such that $v_{k} \neq 0$ :

$$
\sum_{\beta \in \mathbb{Z}} i \beta \int \phi_{\beta}^{(0)}\left(J^{\prime}\right) F_{0}^{\prime}\left(J^{\prime}\right) c_{k, \beta}^{*}\left(J^{\prime}\right) d J^{\prime}=0 .
$$

Generically, this requires $\phi_{\alpha}^{(0)}=0$, for all $\alpha \neq 0$. Hence $\phi^{(0)}(\theta, J)$ has only an $\alpha=0$ component, and $\phi_{0}^{(0)}(J)$ is undetermined at this stage. We now look for $\phi^{(1)}$ such that $\mathcal{L}^{\dagger} \cdot \phi^{(1)}=\phi^{(0)}$. We obtain

$$
\begin{gathered}
\alpha=0: \quad-\sum_{k \in \mathbb{Z}} v_{k} Z_{k}\left[\phi^{(1)}\right] c_{k, 0}(J)=\phi_{0}^{(0)}(J), \\
\alpha \neq 0: \quad \phi_{\alpha}^{(1)}(J)=\frac{1}{i \alpha \Omega_{0}(J)} \sum_{k \in \mathbb{Z}} v_{k} Z_{k}\left[\phi^{(1)}\right] c_{k, \alpha}(J),
\end{gathered}
$$

with

$$
Z_{k}[h]=2 \pi \sum_{\beta \in \mathbb{Z}} i \beta \int h_{\beta}\left(J^{\prime}\right) F_{0}^{\prime}\left(J^{\prime}\right) c_{k, \beta}^{*}\left(J^{\prime}\right) d J^{\prime} .
$$

Equation (A22) is rewritten as

$$
\forall k \in \mathbb{Z}, \quad \sum_{l \in \mathbb{Z}} \Lambda_{k l} Z_{l}\left[\phi^{(1)}\right]=0,
$$

where the $\Lambda$ infinite matrix has been introduced in (A10); (A24) then has a nontrivial solution $\left(y_{k}\right)$. Then (A21) fixes the previously undetermined $\phi_{0}^{(0)}(J) . \phi_{0}^{(1)}(J)$ is a priori undetermined.

We now look for $\phi^{(2)}$ such that $\mathcal{L}^{\dagger} \cdot \phi^{(2)}=\phi^{(1)}$. We obtain

$$
\begin{aligned}
\alpha=0: \quad-\sum_{k \in \mathbb{Z}} v_{k} Z_{k}\left[\phi^{(2)}\right] c_{k, 0}(J)=\phi_{0}^{(1)}(J), & \\
\alpha \neq 0: \quad \phi_{\alpha}^{(2)}(J)= & \frac{1}{i \alpha \Omega_{0}(J)} \sum_{k \in \mathbb{Z}} v_{k} Z_{k}\left[\phi^{(2)}\right] c_{k, \alpha}(J) \\
& +\frac{1}{\left[i \alpha \Omega_{0}(J)\right]^{2}} \sum_{k \in \mathbb{Z}} v_{k} y_{k} c_{k, \alpha}(J) .
\end{aligned}
$$

Using (A5), (A26) is rewritten as

$$
\forall k \in \mathbb{Z}, \quad \sum_{l \in \mathbb{Z}} \Lambda_{k l} Z_{l}\left[\phi^{(2)}\right]=0 .
$$

We may choose the solution $\forall l, Z_{l}\left[\phi^{(2)}\right]=0$. Equation (A25) then implies that $\phi_{0}^{(1)}=0 . \phi_{0}^{(2)}$ is still undetermined at this stage: it can be fixed by orthogonality to the eigenvectors (A20); this yields in particular $\phi_{0}^{(2)} \propto C_{0}(J)$ in the HMF case.

We can now summarize the (generalized) eigenvectors for the nontrivial eigenvalue 0 of $\mathcal{L}^{\dagger}$ :

$$
\begin{aligned}
\phi^{(0)} & =\left(\begin{array}{c}
\sum_{l \in \mathbb{Z}} v_{l} y_{l} c_{l, 0}(J) \\
0
\end{array}\right), \\
\phi^{(1)} & =\left(\begin{array}{c}
0 \\
\frac{1}{i \alpha \Omega_{0}(J)} \sum_{l \in \mathbb{Z}} v_{l} y_{l} c_{l, \alpha}(J)
\end{array}\right), \\
\phi^{(2)} & =\left(\begin{array}{c}
\phi_{0}^{(2)}(J) \\
\frac{1}{\left[i \alpha \Omega_{0}(J)\right]^{2}} \sum_{l \in \mathbb{Z}} v_{l} y_{l} c_{l, \alpha}(J)
\end{array}\right) .
\end{aligned}
$$

The inner products between the (generalized) eigenvectors of $\mathcal{L}$ and of $\mathcal{L}^{\dagger}$ can be computed as (46). We can choose $\phi_{0}^{(2)}$ such that $\left\langle\phi^{(2)}, \psi^{(2)}\right\rangle=0$. Thus, after normalization, the projection operator $\Pi$ on the subspace $\operatorname{Span}\left\{\psi^{(0)}, \psi^{(1)}, \psi^{(2)}\right\}$ is written as (48).

We emphasize once more the $\Omega_{0}(J)^{k}$ factors in the denominators of the expressions of the $\psi^{(j)}$ and $\phi^{(j)}: \Omega_{0}(J)$ may have a zero, however, in the neighborhood of a generic separatrix at action $J_{s}$, it will behave as $1 / \ln \left|J-J_{s}\right|$; as a consequence, all inner products $\left\langle\phi^{(j)}, \psi^{(l)}\right\rangle$ involve only well-defined $J$ integrals.

\section{APPENDIX B: ANALYTIC SOLUTION OF THE REDUCED DYNAMICS}

We derive the explicit solution $Q(t)$ for the reduced dynamics Eq. (59) in terms of the Weierstrass elliptic function $[45,46]$. First using that $Z(t)=Z(0)$ is constant and $\dot{Q}=P$, we get

$$
\ddot{Q}=Z+\mu a Q+\frac{1}{2} r Q^{2},
$$

which, by simple manipulation, change of timescale $T=$ $\sqrt{-r / 12} t$, and $y=-(Q+\mu a / r)$, can be cast into

$$
\left(\frac{d y}{d T}\right)^{2}(T)=4 y^{3}(T)-g_{2} y(T)-g_{3},
$$

with

$$
\begin{aligned}
g_{2}= & \frac{12}{r^{2}}\left[(\mu a)^{2}-2 r Z\right] \\
g_{3}= & \frac{12}{r^{2}}\left\{\left[\frac{\mu a}{r}+Q(0)\right]\left[(\mu a)^{2}-2 r Z\right]\right. \\
& \left.-\frac{r^{2}}{3}\left[\frac{\mu a}{r}+Q(0)\right]^{3}+r P(0)\right\} .
\end{aligned}
$$

Equation (B2) is sometimes used as a definition for the doubly periodic Weierstrass elliptic $\wp$ function and has for solution $y(T)=\wp\left(T-T_{0} ; g_{2}, g_{3}\right)$ where $T_{0}$ is determined by 
the initial conditions $[45,46]$. Hence,

$$
Q(t)=-\left[\wp\left(\sqrt{\frac{-r}{12}} t-T_{0} ; g_{2}, g_{3}\right)+\frac{\mu a}{r}\right] .
$$

In our simulations $P(0)=0, g_{2} \gg g_{3}$ and $r<0$, one can show that $T_{0}=\omega_{1}+\omega_{2}$ where $\omega_{1}, \omega_{2}$ are the two half periods of the $\wp$ function associated with $g_{2}$ and $g_{3}$. When $g_{2}>0$ (corresponding for our numerical experiment to $\varepsilon>0$ ) the oscillation period of $Q(t)$ is asymptotically

$$
\tau=2 \omega_{1} \sqrt{-\frac{12}{r}} \sim \frac{\Gamma(1 / 4)}{\Gamma(3 / 4)} \frac{3^{1 / 4} \sqrt{\pi}}{\left[(\mu a)^{2}-2 r Z\right]^{1 / 4}}
$$

(in this case $\omega_{2}$ is purely imaginary and has no clear physical meaning). Moreover in that case $Q_{\max } \sim$ $-\left\{\sqrt{3\left[(\mu a)^{2}-r Z\right]}+\mu a\right\} / r$. When $Z \ll \mu^{2}$, e.g., when the perturbation is taken exactly along $\psi^{(0)}$ then $Z=0$, we have $Q_{\max } \propto \mu$ so that $Q_{\max } \propto \lambda^{2}$. When $g_{2}<0$ (i.e., $\varepsilon<0$ ), the function $\wp_{(}\left(T-T_{0}\right)$ despite still being periodic encounters a pole in finite time and thus no longer has physical meaning.

\section{APPENDIX C: OBTENTION OF THE NORMAL FORM FOR THE REDUCED HAMILTONIAN}

The procedure to simplify a Hamiltonian in order to obtain a normal form is classical. For self-consistency, we give here more details on how to use it to obtain Hamiltonian (73).

We consider the normal form of the noncanonical Hamiltonian system

$$
\frac{d u}{d t}=\mathbf{J} \nabla_{u} H(u, \mu), \quad u=\left(\begin{array}{l}
u_{0} \\
u_{1} \\
u_{2}
\end{array}\right),
$$

around the origin $u=0$, which is assumed to be stationary. The matrix $\mathbf{J}$ is defined in (60b). From the stationarity of the origin and the use of the Casimir invariant $u_{2}$, we may assume $\nabla_{u} H(0,0)=0$.

The idea to obtain the normal form is to simplify $H(u, \mu)$ by using the coordinate transform

$$
u=T(U),
$$

which gives the transformed Hamiltonian

$$
\bar{H}(U, \mu):=H(T(U), \mu) .
$$

To keep the Poisson structure expressed by the $\mathbf{J}$ matrix, we introduce the constraint

$$
[D T(U)]^{-1} \mathbf{J}[D T(U)]^{-\mathrm{T}}=\mathbf{J}
$$

for the transform $T$, where $D T(U)$ is the Jacobian matrix of $T(U)$ and the superscript $-\mathrm{T}$ represents the transposition of the inverse matrix. Under the constraint (C4), the transformed equation of motion is written as

$$
\frac{d U}{d t}=\mathbf{J} \nabla_{U} \bar{H}(U, \mu), \quad U=\left(\begin{array}{c}
U_{0} \\
U_{1} \\
U_{2}
\end{array}\right) .
$$

We compute the normal form $\bar{H}(U, \mu)$ up to the cubic order of $U$. For this purpose, we expand the transform as

$$
T(U)=U+T_{2}(U)+O\left(|U|^{3}\right),
$$

the original Hamiltonian as

$$
H(u, \mu)=H_{2}(u, \mu)+H_{3}(u, \mu)+O\left(|u|^{4}\right)
$$

from $\nabla_{u} H(0,0)=0$, and the transformed Hamiltonian as

$$
\bar{H}(U, \mu)=\bar{H}_{2}(U, \mu)+\bar{H}_{3}(U, \mu)+O\left(|U|^{4}\right) .
$$

Substituting (C6) into (C7), we have

$$
\bar{H}_{2}(U, \mu)=H_{2}(U, \mu)
$$

and

$$
\bar{H}_{3}(U, \mu)=H_{3}(U, \mu)+\nabla_{u} H_{2}(U, \mu) \cdot T_{2}(U) .
$$

Our job is to simplify $\bar{H}_{3}(U, \mu)$ for a given $H_{2}(u, \mu)$ by using the transform $T_{2}$ under the constraint of

$$
\left(D T_{2}\right) \mathbf{J}-\left[\left(D T_{2}\right) \mathbf{J}\right]^{\mathrm{T}}=0_{3,3},
$$

which comes from (C4) and $0_{3,3}$ is the zero matrix of size 3 .

We first determine the explicit form of $T_{2}(U)$. The general form of $T_{2}(U)$ is written as

$$
T_{2}(U)=\left(\begin{array}{l}
a_{1} U_{0}^{2}+a_{2} U_{1}^{2}+a_{3} U_{2}^{2}+a_{4} U_{0} U_{1}+a_{5} U_{1} U_{2} \\
+a_{6} U_{2} U_{0} \\
b_{1} U_{0}^{2}+b_{2} U_{1}^{2}+b_{3} U_{2}^{2}+b_{4} U_{0} U_{1}+b_{5} U_{1} U_{2} \\
+b_{6} U_{2} U_{0} \\
c_{1} U_{0}^{2}+c_{2} U_{1}^{2}+c_{3} U_{2}^{2}+c_{4} U_{0} U_{1}+c_{5} U_{1} U_{2} \\
+c_{6} U_{2} U_{0}
\end{array}\right),
$$

where $a_{j}, b_{j}$, and $c_{j}$ are real parameters respectively for the first, second, and third elements of the three-dimensional vector $T_{2}(U)$. The constraint $(\mathrm{C} 11)$ requires

$$
\begin{aligned}
& \left(2 a_{1}+b_{4}\right) U_{0}+\left(a_{4}+2 b_{2}\right) U_{1}+\left(a_{6}+b_{5}\right) U_{2}=0 \\
& 2 c_{1} U_{0}+c_{4} U_{1}+c_{6} U_{2}=0 \\
& 2 c_{2} U_{1}+c_{4} U_{0}+c_{5} U_{2}=0
\end{aligned}
$$

and, therefore,

$$
T_{2}(U)=\left(\begin{array}{l}
a_{1} U_{0}^{2}+a_{2} U_{1}^{2}+a_{3} U_{2}^{2}+a_{4} U_{0} U_{1}+a_{5} U_{1} U_{2} \\
+a_{6} U_{2} U_{0} \\
b_{1} U_{0}^{2}+b_{2} U_{1}^{2}+b_{3} U_{2}^{2}+b_{4} U_{0} U_{1}+b_{5} U_{1} U_{2} \\
+b_{6} U_{2} U_{0} \\
c_{3} U_{2}^{2}
\end{array}\right)
$$

with

$$
b_{2}=-\frac{a_{4}}{2}, \quad b_{4}=-2 a_{1}, \quad b_{5}=-a_{6} .
$$

Now, $a_{1}, \ldots, a_{6}, b_{1}, b_{3}, b_{6}$ and $c_{3}$ are the free parameters. The simplification of $\bar{H}_{3}(U, \mu),(\mathrm{C} 10)$, is realized by eliminating terms of $H_{3}(U, \mu)$ by using these free parameters included in $\nabla_{u} H_{2}(U, \mu) \cdot T_{2}(U)$. 
The next step is to determine the quadratic Hamiltonian $H_{2}(u, \mu)$. We set

$$
H_{2}(u, \mu)=\frac{u_{1}^{2}-\mu u_{0}^{2}}{2}-u_{0} u_{2},
$$

which gives the linear part as

$$
\mathbf{J} \nabla_{u} H_{2}(u, \mu)=\left(\begin{array}{ccc}
0 & 1 & 0 \\
\mu & 0 & 1 \\
0 & 0 & 0
\end{array}\right)\left(\begin{array}{l}
u_{0} \\
u_{1} \\
u_{2}
\end{array}\right) .
$$

Thus, the choice of (C16) permits to have the threedimensional Jordan block at $\mu=0$, which is predicted in the general setting, and to change stability of the origin at $\mu=0$ (stable for $\mu<0$ and unstable for $\mu>0$ ).

Before computing the normal form, we remark that $|\mu|$ is small and the dominant part of $\bar{H}_{3}(U, \mu)$ is given by $\bar{H}(U, 0)$. Therefore, we use $\nabla_{u} H_{2}(U, 0) \cdot T_{2}(U)$ to eliminate terms of $H_{3}(U, 0)$. The quadratic Hamiltonian $H_{2}(u, \mu)$, (C16), with the transform $T_{2}(U),(\mathrm{C} 14)$, give

$$
\begin{aligned}
\nabla_{u} & H_{2}(U, 0) \cdot T_{2}(U) \\
= & \left(\frac{-a_{4}}{2}\right) U_{1}^{3}+\left(-a_{3}\right) U_{2}^{3}+\left(-a_{4}+b_{6}\right) U_{0} U_{1} U_{2} \\
& +b_{1} U_{0}^{2} U_{1}-a_{1}\left(U_{0}^{2} U_{2}+2 U_{1}^{2} U_{0}\right)+\left(-a_{2}-a_{6}\right) U_{1}^{2} U_{2} \\
& +\left(-a_{6}-c_{3}\right) U_{2}^{2} U_{0}+\left(-a_{5}+b_{3}\right) U_{2}^{2} U_{1} .
\end{aligned}
$$

Thanks to the 10 free parameters, we can eliminate almost all terms of $H_{3}(U, 0)$, but $U_{0}^{3}$ can not be eliminated. In addition, one of the two terms $U_{0}^{2} U_{2}$ or $U_{1}^{2} U_{0}$ survives since they share the free parameter $a_{1}$.

Finally, we have the normal form of $\bar{H}$ up to the cubic order as

$$
\bar{H}_{\text {norm }}=\frac{U_{1}^{2}-\mu U_{0}^{2}}{2}-U_{0} U_{2}+r U_{0}^{3}+s U_{0}^{2} U_{2}
$$

or

$$
\bar{H}_{\text {norm }}=\frac{U_{1}^{2}-\mu U_{0}^{2}}{2}-U_{0} U_{2}+r U_{0}^{3}+\tilde{s} U_{1}^{2} U_{0},
$$

where $r, s$ or $r, \tilde{s}$ are undetermined parameters. Since $U_{2}$ is a conserved quantity, we see that, upon changing the definition of $\mu$, the term $s U_{0}^{2} U_{2}$ can be removed in (C19), which makes this form easier to work with. Finally, rescaling time and $U_{0}$, it is possible to take $r=1$, and obtain (72).

Introducing the cubic order coordinate transform $T_{3}(U)$ and using the free parameters in $T_{2}(U)$ and $T_{3}(U)$, we find the terms of $U_{0}^{4}$ and $U_{0}^{2} U_{1}^{2}$ in the quartic order normal form, $\bar{H}_{4}$. Depending on their signs, these terms may confine orbits in a bounded region. Notice however that (1) a full reduction of the dynamics up to quartic order would require to compute the curvature of the central manifold, whereas considering its tangent space is enough up to cubic order and (2) while largeamplitude periodic trajectories appearing at quartic order are reminiscent of the numerical results (see inset of Fig. 2), they go beyond the perturbative regime, so that we cannot expect a quantitative agreement between reduced and exact dynamics.
[1] P. J. Morrison and R. D. Hazeltine, Hamiltonian formulation of reduced magnetohydrodynamics, Noncanonical Hamiltonian Density Formulation of Hydrodynamics and Ideal Magnetohydrodynamics, Phys. Fluids 27, 886 (1984).

[2] P. J. Morrison and J. M. Greene, Noncanonical Hamiltonian Density Formulation of Hydrodynamics and Ideal Magnetohydrodynamics, Phys. Rev. Lett. 45, 790 (1980); 48, 569(E) (1982).

[3] P. J. Morrison, The Maxwell-Vlasov equations as a continuous Hamiltonian system, Phys. Lett. A 80, 383 (1980).

[4] P. J. Morrison, Hamiltonian description of the ideal fluid, Rev. Mod. Phys. 70, 467 (1998).

[5] L. Landau, On the vibration of the electronic plasma, J. Phys. USSR 10, 25 (1946); in Collected Papers of L. D. Landau, edited by D. T. Haar (Pergamon Press, Oxford, 1965).

[6] K. R. Meyer, Normal forms for Hamiltonian systems, Celest. Mech. Dyn. Astron. 9, 517 (1974).

[7] K. R. Meyer, Generic bifurcations in Hamiltonian systems, in Dynamical Systems-Warwick 1974, edited by A. Manning (Springer, Berlin, 1975), pp. 62-70.

[8] M. Golubitsky and I. Stewart with an Appendix by J. Marsden, Generic bifurcation of Hamiltonian systems with symmetry, Physica D 24, 391 (1987).

[9] J. Xiao, J. Liu, H. Qin, and Z. Yu, A variational multisymplectic particle-in-cell algorithm with smoothing functions for the Vlasov-Maxwell system, Phys. Plasmas 20, 102517 (2013).
[10] B. A. Shadwick, A. B. Stamm, and E. G. Evstatiev, Variational formulation of macro-particle plasma simulation algorithms, Phys. Plasmas 21, 055708 (2014).

[11] P. J. Morrison, Structure and structure-preserving algorithms for plasma physics, Phys. Plasmas 24, 055502 (2016).

[12] J. W. Burby, Finite-dimensional collisionless kinetic theory, Phys. Plasmas 24, 032101 (2017).

[13] M. Kraus, K. Kormann, P. J. Morrison, and E. Sonnendrücker, GEMPIC: Geometric electromagnetic particle-in-cell methods, J. Plasma Phys. 84, 905830401 (2017).

[14] K. Modin and M. Viviani, Lie-Poisson methods for isospectral flows, Found. Comput. Math. 20, 889 (2020).

[15] K. Modin and M. Viviani, A Casimir preserving scheme for long-time simulation of spherical ideal hydrodynamics, J. Fluid Mech. 884, A22 (2020).

[16] P. J. Morrison and J. Vanneste, Weakly nonlinear dynamics in noncanonical Hamiltonian systems with applications to fluids and plasmas, Ann. Phys. 368, 117 (2016).

[17] J. Dawson, On Landau damping, Phys. Fluids 4, 869 (1961).

[18] E. Frieman, S. Bodner, and P. Rutherford, Some new results on the quasi-linear theory of plasma instabilities, Phys. Fluids 6, 1298 (1963).

[19] A. Simon and M. N. Rosenbluth, Single-mode saturation of the bump-on-tail instability: Immobile ions, Phys. Fluids 19, 1567 (1976). 
[20] J. Denavit, Simulations of the single-mode, bump-on-tail instability, Phys. Fluids 28, 2773 (1985).

[21] J. D. Crawford, Universal Trapping Scaling on the Unstable Manifold for a Collisionless Electrostatic Mode, Phys. Rev. Lett. 73, 656 (1994).

[22] J. D. Crawford, Amplitude Equations for Electrostatic Waves: Universal Singular Behavior in the Limit of Weak Instability, Phys. Plasmas 2, 97 (1995).

[23] N. J. Balmforth, P. J. Morrison, and J.-L. Thiffeault, Pattern formation in Hamiltonian systems with continuous spectra; A normal-form single-wave model, arXiv:1303.0065.

[24] T. M. O’Neil, J. H. Winfrey, and J. H. Malmberg, Nonlinear interaction of a small cold beam and a plasma, Phys. Fluids 14, 1204 (1971).

[25] D. del-Castillo-Negrete, Nonlinear evolution of perturbation in marginally stable plasmas, Phys. Lett. A 241, 99 (1998).

[26] J. D. Crawford and A. Jayaraman, Nonlinear Saturation of an Electrostatic Wave: Mobile Ions Modify Trapping Scaling, Phys. Rev. Lett. 77, 3549 (1996).

[27] J. D. Crawford and A. Jayaraman, First principles justification of a "single wave model" for electrostatic instabilities, Phys. Plasmas 6, 666 (1999).

[28] N. J. Balmforth and R. R. Kerswell, Saturation of electrostatic instability in two-species plasma, J. Plasma Phys. 68, 87 (2002).

[29] J. Barré, D. Métivier, and Y. Y. Yamaguchi, Trapping scaling for bifurcations in the Vlasov systems, Phys. Rev. E 93, 042207 (2016).

[30] C. Pichon and D. Lynden-Bell, Bar instabilities in galaxies, in Statistical Description of Transport in Plasma, Astro- and Nuclear Physics, edited by J. Misquich, G. Pelletier, and P. Schuck (Nova Science Publisher, 1993), p. 261.

[31] S. Inagaki and T. Konishi, Dynamical stability of a simple model similar to self-gravitating systems, Publ. Astron. Soc. Jpn. 45, 733 (1993).

[32] M. Antoni and S. Ruffo, Clustering and relaxation in Hamiltonian long-range dynamics, Phys. Rev. E 52, 2361 (1995).

[33] A. Weinstein, The local structure of Poisson manifolds, J. Diff. Geom. 18, 523 (1983).
[34] J. Barré, A. Olivetti, and Y. Y. Yamaguchi, Dynamics of perturbations around inhomogeneous backgrounds in the HMF model, J. Stat. Mech. (2010) P08002.

[35] T. M. Rocha Filho, Solving the Vlasov equation for onedimensional models with long range interactions on a GPU, Comput. Phys. Commun. 184, 34 (2013).

[36] J. Banks and J. Hittinger, A new class of nonlinear finite-volume methods for Vlasov simulation, IEEE Trans. Plasma Sci. 38, 2198 (2010).

[37] D. Silantyev, P. Lushnikov and H. Rose, Langmuir wave filamentation in the kinetic regime. I. Filamentation instability of Bernstein-Greene-Kruskal modes in multidimensional Vlasov simulations, Phys. Plasmas 24, 042104 (2017).

[38] S. Pankavich and R. Allen, Instability conditions for some periodic BGK waves in the Vlasov-Poisson system, Eur. Phys. J. D 68, 363 (2014).

[39] J. Binney and S. Tremaine, Galactic Dynamics, 2nd ed. (Princeton University Press, Princeton, 2008).

[40] P. L. Palmer, J. Papaloizou, and A. J. Allen, Neighbouring equilibira to radially anisotropic spheres-Possible end-states for violently relaxed stellar systems, Mon. Not. R. Astron. Soc. 246, 415 (1990).

[41] V. L. Polyachenko, E. V. Polyachenko, and I. G. Shukhman, Gravitational loss-cone instability, J. Exp. Theor. Phys. 104, 396 (2007).

[42] P. Smereka, Synchronization and relaxation for a class of globally coupled Hamiltonian systems, Physica D 124, 104 (1998).

[43] G. Russo and P. Smereka, Kinetic theory for bubbly flow I: Collisionless case, SIAM J. Appl. Math. 56, 327 (1996).

[44] P. Smereka, A Vlasov equation for pressure wave propagation in bubbly fluids, J. Fluid Mech. 454, 287 (2002).

[45] J. Snape, Applications of Elliptic Functions in Classical and Algebraic Geometry (University of Durham, 2004).

[46] G. Pastras, Four lectures on Weierstrass elliptic function and applications in classical and quantum mechanics, arXiv:1706.07371. 